\title{
Féeries
}

Études sur le conte merveilleuX, XVII ${ }^{e}$ XIXe siècle

$9 \mid 2012$

Le dialogisme intertextuel des contes des Grimm

\section{Le dialogue intertextuel du Trésor des Contes d'Henri Pourrat avec les Grimm et Perrault}

Intertextual dialogue between Henri Pourrat'sTrésor des contes, the Grimms and Perrault

Jean-Michel Adam

\section{CpenEdition}

Journals

Édition électronique

URL : http://journals.openedition.org/feeries/827

DOI : $10.4000 /$ feeries.827

ISSN : 1957-7753

Éditeur

UGA Éditions/Université Grenoble Alpes

Édition imprimée

Date de publication : 15 octobre 2012

Pagination : 161-196

ISBN : 978-2-84310-233-2

ISSN : 1766-2842

\section{Référence électronique}

Jean-Michel Adam, «Le dialogue intertextuel du Trésor des Contes d'Henri Pourrat avec les Grimm et Perrault », Féeries [En ligne], 9 | 2012, mis en ligne le 15 octobre 2013, consulté le 08 septembre 2020. URL : http://journals.openedition.org/feeries/827 ; DOI : https://doi.org/10.4000/feeries.827

\section{(c) Féeries}


Jean-Michel Adam

Centre de recherche en langues et littératures européennes comparées (CLE),

Université de Lausanne

\title{
LE DIALOGUE INTERTEXTUEL DU TRÉSOR DES CONTES D'HENRI POURRAT AVEC LES GRIMM ET PERRAULT
}

\author{
Reste que les contes populaires doivent être \\ d'origine plus lettrée qu'on ne le dirait d'abord. \\ Henri Pourrat, Ambert, I8 octobre $1946^{\mathrm{I}}$.
}

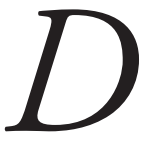

ANS LA PERSPECTIVE de la présente livraison de Féeries, centrée sur le dialogisme intertextuel des contes ${ }^{2}$, j'ai choisi de constituer un corpus de douze textes autour de quatre contes du Trésor des contes d'Henri Pourrat : Le conte de Marie-Cendron (Livre I, I948), Le conte du Chaperon rouge (Livre II, I949), Le conte de la Belle au bois dormant (Livre IV, I953) et Le conte de la Barbe-Bleue (Livre VIII, I957). Ces quatre contes présentent l'intérêt de dialoguer intertextuellement à la fois avec quatre contes de Perrault : Cendrillon ou la petite pantoufle de verre, Le Petit Chaperon rouge, La Belle au bois dormant et La Barbe bleüe des Histoires ou Contes du temps passé. Avec des Moralitez (I697), et avec quatre contes des Grimm (I8I2-I857) : Aschenputtel, Rotkäppchen, Dornröschen et Blaubart (contes $\mathrm{n}^{\text {os }} 2 \mathrm{I}, 26$, 50 et 62 de l'édition de I8I2 des Kinder- und Hausmärchen; ci-après $K H M$ ).

I. H. Pourrat, "Note de l'auteur», en introduction au Trésor des contes. Je citerai autant que possible les textes et notes de H. Pourrat dans l'édition «Omnibus» (2009) de B. Bricout. Les trois premiers contes seront toujours cités dans la pagination du volume I et le quatrième dans celle du second volume. L'indication du numéro du volume et de la page évitera ainsi les nombreux renvois en note de bas de page. Les textes de Perrault seront donnés dans le texte du fac-similé de la première édition (I697), citée en bibliographie; je ne normaliserai que les graphèmes «f $f$ valant pour " $\mathrm{s}$ » et respecterai l'orthographe d'époque. Pour les Grimm, l'édition I857 des Kinder- und Hausmärchen sera utilisée pour les trois premiers contes et celle de I8I2 pour Blaubart; toutes les traductions sont miennes, avec l'aide précieuse de U. Heidmann.

2. Je reprends le terme introduit par U. Heidmann dans son article introductif et exemplifié dans la première partie de Textualité et intertextualité des contes (U. Heidmann et J.-M. Adam, 20IO).

Féeries, nº 9 , 2012, p. I6I-I96.

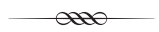


Parlant de son corpus de "contes français», H. Pourrat écrit :

Au vrai, ils sont à toute la vieille paysannerie d'Europe et d'ailleurs. Marie-Cendron contée par $\mathrm{M}^{\mathrm{lle}}$ Alphonsine Dapzol, couturière au bourg de Champétières, est beaucoup plus proche de la Cendrillon des Grimm que de celle de Perrault; et $\mathrm{M}^{\text {lle }}$ Dapzol tient ce conte de sa mère qui le tenait de vieilles personnes du pays. D'autres femmes de campagne le récitent sous cette même forme d'après des tantes ou des grand-mères qu'on imagine mal ayant jamais lu Grimm. Sait-on? Il suffirait d'un recueil du Journal des Enfants, vers I840, passant de la maison du maître à la métairie... Cependant, ces versions qui tiennent de Grimm et en diffèrent aussi, semblent bien plus anciennes en France que les premières traductions publiées ${ }^{3}$.

Si la Cendrillon racontée par Alphonsine Dapzol, 52 ans, couturière de Champétières, dans une lettre d'octobre 1943, est proche des Grimm, Antoinette Rocher, 75 ans, de Pacros, déclare ouvertement, en 1936, qu'elle va raconter cette histoire "comme dans Perrault ${ }^{4}$ ». Le texte publié par H. Pourrat intègre également le récit fait en I9I2 par Marie Claustre, 78 ans, dentellière à Marsan. H. Pourrat aurait pu également prendre l'exemple du Conte du Chaperon rouge qui est, par sa fin heureuse et d'autres détails sur lesquels je reviendrai, plus proche du texte des Grimm que de Perrault, mais il est vrai que son travail de "restauration" du conte a été fait à partir de textes publiés, probablement les mêmes que ceux que Paul Delarue utilise pour émettre l'hypothèse anachronique que nous critiquons dans Textualité et intertextualité des contes'. À l'inverse, Le conte de la Belle au bois dormant semble si proche de Perrault que Gilbert Rouger a pu le qualifier de «médiocre amplification du récit de Perrault ${ }^{6}$ », jugement de valeur qu'il nous faudra tempérer. M. Claustre a raconté à H. Pourrat, en I9I2, un Conte de la Barbe-Bleue qui mêle manifestement La Barbe bleüe de Perrault et le Blaubart des Grimm. Mais on trouve aussi un Conte d'Isabelle et de ses trois frères dont la deuxième partie est très proche de La Barbe bleüe de Perrault et un Front d'airain, véritable histoire de brigands plus proche, quoi qu'en

3. H. Pourrat, Le Trésor des contes, volume I, 2009, p. I2-I3. On doit effectivement tenir compte de la publication, en 1869 , de la traduction, au titre imitant celui de Perrault, des Contes allemands du temps passé (F. Frank et E. Alsleben, traducteurs, Paris, Didier). Parmi les contes traduits (Blanche Neige, L'eau de la vie, La Dame Hollé, La lumière bleue, Raiponce, ou encore La princesse sur les pois), il y a effectivement Cendrillon et Les trois nains de la forêt.

4. Informations données dans le livre important de B. Bricout, Le Savoir et la Saveur, 1992, p. 260. L'auteur se livre à une lecture ethno-littéraire de deux des textes dont je vais amorcer ici une analyse toute différente sur le fond: Le conte de Marie-Cendron (p. 260-285) et Le conte du Chaperon rouge (p. 304-328).

5. U. Heidmann et J.-M. Adam, ouvr. cité, p. 2I-25.

6. G. Rouger, Contes de Perrault, Paris, Garnier, 1967, p. 96. 
dise H. Pourrat au début de sa «Note de l'auteur» de 1946, du Mordschloss (Le château des meurtres) des Grimm que du texte de Perrault.

Les textes rassemblés par $\mathrm{H}$. Pourrat proviennent autant de conteurs des monts du Forez et du Livradois que de revues, journaux, almanachs, recueils de traditions populaires locales, et même de rédactions d'élèves. Ces contes sont explicitement récrits par un écrivain reconnu, enrôlé un temps, en 1943, dans le projet culturel de Vichy, mais heureusement resté en dehors de la dérive antisémite du régime, comme le précise Bernadette Bricout $^{7}$. Je m'intéresserai à quatre contes qui re-racontent des textes de Perrault et des Grimm pour les faire signifier autrement, dans une autre ère culturelle : l'Auvergne de la première moitié du $\mathrm{xx}^{\mathrm{e}}$ siècle, bien éloignée des salons de la France de la fin du règne de Louis XIV et de l'Allemagne protestante de la première moitié du XIX ${ }^{\mathrm{e}}$ siècle. L'hypothèse de travail sur laquelle je m'appuie est proche de celle que définit ici-même, dans son article introductif, Ute Heidmann, à savoir le fait que le dialogue intertextuel des contes atteste la présence du souvenir de contes écrits transportés et reconfigurés de bouche à oreille, et retournés à l'écrit au fil du temps et des déplacements de populations.

Comme Charles Ferdinand Ramuz et Jean Giono, H. Pourrat participe au débat qui divise les écrivains régionalistes «entre "folkloristes" et "vrais" écrivains de l'humain ${ }^{8}$ ». H. Pourrat a beaucoup dialogué, entre 1924 et I926, avec Ch. F. Ramuz qu'il admirait, mais reste-t-il plus proche du courant régionaliste, soucieux du documentaire fidèle et de l'authentique que du courant esthétique du «grand style paysan» prôné par Ch. F. Ramuz, comme le pense Jérôme Meizoz?

[...] L'évolution de Ramuz tend à conquérir le prestige formel (critère de littérarité) et métaphysique (critère d'universalité) en demeurant dans une zone peu noble quant à la thématique (le roman rustique); elle représente un compromis délicat et novateur?.

En examinant de près l'écriture de quatre textes du Trésor des contes, nous verrons que ce n'est peut-être pas aussi simple et qu'une écriture de l'oralité et même de la vocalité émerge, même si elle reste en deçà de l'ampleur des audaces stylistiques d'un Ramuz.

7. «Il pourra dans le même temps défendre ce qu'il croit être le choix qui s’impose aux Français et protéger les Juifs des rafles allemandes.» (B. Bricout, ouvr. cité, p. 387 ).

8. J. Meizoz, L'àge du roman parlant, 200I, p. 63.

9. Ibid., p. 64. 


\section{Le Trésor des contes : une œuvre au cœur des débats}

L'œuvre de H. Pourrat est intéressante en raison de son positionnement à la fois dans un débat avec la folkloristique de van Gennep, des Grimm, d'Aarne-Thompson et de Delarue, et dans les débats littéraires de la France des années 1920 à l'après seconde guerre mondiale. H. Pourrat comprend la méthodologie ethnographique des folkloristes Arnold van Gennep et Paul Delarue, mais il critique la transcription plate et supposée objective et scientifique de ce dernier dans des termes qui éclairent son propre projet : "Il a trop tenu, je crois, à une authenticité proche des conteurs, ce qui fait que pour la masse ces contes restent déroutants et assez illisibles ${ }^{10}$." $\mathrm{La}$ double contrainte imposée est ainsi parfaitement résumée par $\mathrm{H}$. Pourrat : «Impossible de ne pas toucher à ces contes : donnés tels quels, sans sel ni sauge, ils restent curiosité de spécialistes. Et impossible d'y toucher : ils ne sont pas tiens, ce sont des classiques de la paysannerie ${ }^{\text {II }}$.» De façon assez subtile, $\mathrm{H}$. Pourrat redéfinit la notion même de «conte populaire» $:$ «Peutêtre n'y a-t-il pas de contes populaires, si on entend par là contes dus uniquement au peuple? Mais on peut nommer contes populaires ceux qui lui ont parlé, qu’il a adoptés et auxquels il a donné une certaine façon à lui ${ }^{12}$."

Quand il se demande "Comment se fait-il qu'il y ait dans le même canton tant de variantes pour un même conte? Mais il court aussi le Rouergue [...]. Il court le Limousin, il court la Bretagne [...]. Si l'on cherchait, on le trouverait peut-être partout, de l'Écosse au Tyrol ${ }^{13}$ », H. Pourrat reprend ici la fiction philologique constituée par les Grimm et prise à la lettre par les folkloristes, à savoir l'impression d'universalité des contes classés par eux en "contes-types». Définissant les contes comme "une grande entreprise magique», "primordiale et universelle», comme "littérature première» et "universelle tentative d'incantation ${ }^{14} », \mathrm{H}$. Pourrat confère le "prestige métaphysique» du "critère d'universalité » au conte (pour reprendre les termes de J. Meizoz). H. Pourrat écrit en effet très clairement :

Les contes, surtout les contes merveilleux, sont partout les mêmes : sur les papyrus égyptiens et dans les veillées gasconnes ou champenoises; dans tel district du Bengale, aux îles Aran, dans la commune de Valcivières, les mêmes. Partout mêmes histoires, de princesse changée en grenouille, quand ce n'est pas en quenouille ou en fuseau qui

Io. H. Pourrat, lettre non datée à Jean Combe, citée par B. Bricout, ouvr. cité, p. 64.

II. H. Pourrat, La Porte du verger, Uzès, Éditions de la Cigale, I938, p. I44.

I2. H. Pourrat, "Note de l'auteur", Le Trésor des contes, volume I, p. I4.

I3. Note d'H. Pourrat datée de I95I, reprise dans l'introduction à l'édition Folio ( $\left.{ }^{\circ}{ }_{3857}\right)$ du Trésor des contes. Les amours, I98I, p. Io.

I4. Ibid., p. 15-16. 
parle, d'alliances conclues avec la carpe ou avec la fourmi, de plume d'or qui porte malencontre, d'anneau tombé dans le gâteau, ou de tache de sang qui ne s'efface plus. Et ces imaginations déraisonnées de transferts, de langage des bêtes compris, de sorts jetés ou de chances inouïes ne sont pas arabes ou auvergnates, tartares ou incas, mais humaines ${ }^{15}$.

Stratégiquement, H. Pourrat situe de ce fait son entreprise au-delà du régionalisme dans lequel la critique persiste à le cantonner : «Et l'on est ici aux antipodes du régionalisme, car il semble qu'il y ait une unité de l'esprit humain, que cette tradition obscure, de magie, de mythologie et de poésie soit à peu près partout de même chiffre ${ }^{16}$." Si une histoire apparaît dans des formes semblables dans des cultures différentes, on en déduit que l'humanité est la même et on oublie le fait que cette unité mythique n'est possible qu'à l'aune d'un préconstruit idéologique, source des colonialismes militaires et religieux, puis du triomphe mondialiste du libéralisme économique et politique.

La théorie de la comparaison différentielle élaborée par U. Heidmann, centrée tout au contraire sur l'altérité — au sens fort de ce qui rend un texte autre - et la différenciation comme principe constitutif, est une poétique du divers (pour reprendre l'expression d'Édouard Glissant ${ }^{17}$ ). Elle s'intéresse à ce qui change et produit ainsi du sens toujours nouveau. Dans la langue des quatre contes de $\mathrm{H}$. Pourrat dont j'ai choisi de parler, mélange d'oralité du contage et de français littéraire, nous pouvons encore entendre les langues et les histoires mêlées des textes de Perrault et des Grimm, ce qui me paraît plus intéressant que la mise en évidence d'un abstrait humain universellement le même et qui se réduirait à des prétendus "contes-types». Nous verrons qu'il y a de l'autre dans le conte, qu'il y a du français de l'âge classique et de l'allemand de l'époque romantique, comme il y avait encore du latin ("Anna soror», "Anna vides») dans "Anne ma sœur Anne, ne vois-tu rien venir?» de La Barbe bleüe de Perrault (sans parler de la présence massive du texte du conte de Psyché d'Apulée, comme U. Heidmann l'a démontré, dans les contes de Perrault ${ }^{18}$ ), et comme il y a du Perrault dans les quatre contes des Grimm étudiés ici.

Pour soutenir linguistiquement cette approche discursive et différentielle, je citerai une des plus intéressantes notes manuscrites de Ferdinand de Saussure. Le linguiste genevois a consacré aux Nibelungen et à la légende

I5. Ibid., p. I6.

16. H. Pourrat, La ligne verte, Paris, Gallimard, 1929, p. 62-62.

17. É. Glissant, Introduction à une poétique du divers, 1996.

I8. U. Heidmann, dans U. Heidmann et J.-M. Adam, ouvr. cité, 20IO, p. 3I-I52. 
de Tristan et Iseut, entre I903 et I9IO, 8I4 feuillets (la plupart recto verso) de cahiers d'écolier qui constituaient le chantier d'un véritable projet de livre sur les légendes germaniques :

Ce qui fait la noblesse de la légende comme de la langue, c'est que condamnées l'une et l'autre à ne se servir que d'éléments apportés devant elles et d'un sens quelconque, elles les réunissent et en tirent continuellement un sens nouveau. [...]

[...] Car étant donnés cinq ou six éléments matériels, le sens changera dans l'espace de quelques minutes si je les donne à combiner à cinq ou six personnes travaillant séparément ${ }^{19}$.

Ces lignes font pleinement sens quand on rapproche ce que dit F. de Saussure des "éléments matériels» de la façon dont il parle des signes linguistiques dans la «note sur le discours»:

[...] Que faut-il pour que nous ayons l'idée qu'on veut signifier quelque chose, en usant de termes qui sont à disposition dans la langue? C'est la même question que de savoir ce qu'est le discours, et à première vue la réponse est simple : le discours consiste, fût-ce rudimentairement et par des voies que nous ignorons, à affirmer un lien entre deux des concepts qui se présentent revêtus de la forme linguistique, pendant que la langue ne fait préalablement que réaliser des concepts isolés, qui attendent d'être mis en rapport entre eux pour qu'il y ait signification de pensée ${ }^{20}$.

Il ressort clairement de ces deux citations que, pour F. de Saussure luimême, le sens est le produit variable d'une mise en œuvre combinatoire qui vaut autant pour les «symboles» que sont les signes de la langue, que pour les «symboles» constitutifs de textes narratifs comme les légendes et les contes : «Ils font tous partie de la sémiologie $e^{21}$ ». F. de Saussure énumère les unités textuelles qui gravitent autour des symboles narratifs centraux que sont les personnages :

Chacun des personnages est un symbole dont on peut voir varier $[\ldots]-$ a) le nom, b) la position vis-à-vis des autres, c) le caractère, d) la fonction, les actes. Si un nom est transposé, il peut s'ensuivre qu’une partie des actes sont transposés, et réciproquement, ou que le drame tout entier change par un accident de ce genre ${ }^{22}$.

C'est très précisément cette transposition qui advient quand la Barbe bleüe de Perrault devient le Blaubart des Grimm et plus encore, nous le

I9. F. de Saussure, Ms. fr. 3959-IO, p. I8; «Légendes et récits d'Europe du Nord», 2003, p. 42I-422.

20. Enregistrée sous la cote Ms. fr. 396I (cahier d'écolier sans titre), aujourd'hui accessible dans l'édition des Écrits de linguistique générale, 2002, p. 277. J'en parle plus longuement dans La linguistique textuelle, 20II, p. 19-24, comme j'avais abordé la question des anagrammes dans Linguistique et discours littéraire (J.-M. Adam et J.-P. Goldenstein, 1976, p. 42-59).

2I. F. de Saussure, Ms. fr. 3958-4; «Légendes et récits d'Europe du Nord», 2003, p. 367.

22. Ibid., p. 367-368. 
verrons plus loin, la Barbe-Bleue de H. Pourrat. Quand le Petit Chaperon rouge devient Chaperon rouge, chez $\mathrm{H}$. Pourrat, la configuration familiale change : celle de Perrault n'a pas de parrain bûcheron qui vole à son secours, ni de mère-grand prévoyante, avec laquelle elle vit seule, comme chez $\mathrm{H}$. Pourrat. Dornröschen a la chance de ne pas avoir de belle-mère ogresse qui menace sa vie et celle de ses deux enfants. Aschenputtel reçoit une aide magique toute différente, obtenue à force de dévotion et de larmes versées sur la tombe de sa mère. En paraphrasant F. de Saussure, nous pouvons dire que le drame tout entier change par des accidents de ce genre.

Comme le montre Béatrice Turpin dans la présentation de son édition des cahiers consacrés aux légendes germaniques et à l'histoire de Tristan, le projet de F. de Saussure est discursif : il dégage les légendes de la ressemblance avec les données historiques et s'intéresse aux multiples narrations des histoires de Tristan, de Siegfried et d'autres héros germaniques et scandinaves. De cette pensée de la textualité et du sens comme variation infinie de la composition des éléments, je tire des raisons linguistiques (Saussure dirait sémiologiques) de suivre la théorie de la comparaison différentielle d'U. Heidmann, en étant, pour ma part, attentif aux configurations d'éléments récurrents, ou au moins ressemblants, et surtout aux variations, même les plus infimes, de ces éléments et du sens. Aucun texte de Perrault, des Grimm et de Pourrat n'est pour moi une variante d'une base aussi réduite qu'un "conte-type». Les variations des intrigues et des propriétés des personnages ne seront pas ici traitées structuralement, selon les lois d'une grammaire du conte ${ }^{23}$, mais comme des variations discursives, des variations de textes et de langues inscrits dans des projets discursifs spécifiques. C'est dans les différences entre les textes de Pourrat, des Grimm et de Perrault que je chercherai ce qu'U. Heidmann appelle le «dialogue intertextuel» et les effets de sens de la «reconfiguration" d'un texte par un autre. Ce qui m'intéressera prioritairement, c'est le fait que les quatre contes choisis de $\mathrm{H}$. Pourrat ne reconfigurent pas un des deux textes antérieurs, mais généralement les deux à la fois, dans un double dialogue intertextuel en même temps très proche et très différent des textes antérieurs ${ }^{24}$.

On le perçoit déjà dans le choix des surnoms : Cendrillon se christianise en devenant «Marie-Cendron» et le "Chaperon rouge» de H. Pourrat a perdu l'adjectif diminutif "petit» et acquis l'âge de presque huit ans (vol. I,

23. La Morphologie du conte établit, en effet, une sorte de langue des contes qui expliquerait, selon V. Propp, la «similitude des contes du monde entier» (I970, p. 27).

24. Sur cette forme d'analyse de plusieurs dialogues intertextuels au sein d'un même texte, voir les chap. 2 et 3 d'U. Heidmann, dans Textualité et intertextualité des contes. 
p. 247). On sait que le titre du conte de Perrault joue avec un mot déjà vieilli à la fin du XVII ${ }^{\text {e }}$ siècle. Cette mise à distance de l'emploi référentiel du mot ancien libère le jeu ironique sur l'expression figurée que donne le Dictionnaire de l'Académie : "On appelle figur. Grand chaperon, Les femmes d'âge qui accompagnent les jeunes filles dans les compagnies, par bienséance $\&$ comme pour répondre de leur conduite ${ }^{25}$ " (emploi confirmé par le dictionnaire de Furetière). Quant à la parure que la mère-grand fait faire à sa petite fille, il s'agit d'une bande d'étoffe en velours ou satin posée à plat sur la tête et pendant en arrière. Le Dictionnaire de l'Académie de I694 en parle comme d'une coiffure démodée «que les filles \& les femmes qui n'estoient point Demoiselles, attachoient sur leur teste, il n'y a pas encore long-temps». Le nom de Rotkäppchen, dans le conte des Grimm, porte le souvenir de ce jeu avec la langue qui concentrait toute l'intrigue du conte de Perrault; mais son surnom ne signifie plus la même chose. La traduction allemande du surnom ne reprend pas les connotations du mot "chaperon" et la traduction française la plus juste du mot composé Rotkäppchen serait, dans l'ordre strictement inverse des composantes du surnom allemand, "Petit-bonnet-rouge». Une fois passé dans la mémoire collective, le nom «Petit Chaperon rouge» est accepté en dehors du sens attesté par les dictionnaires du XVII ${ }^{\mathrm{e}}$ siècle. La ressemblance phonique avec capuchon rend l'interprétation du mot ancien et la transposition des Grimm possibles :

Es war einmal eine kleine süße Dirne, die hatte jedermann lieb, der sie nur ansah, am allerliebsten aber ihre Großmutter, die wußte gar nicht, was sie alles dem Kinde geben sollte. Einmal schenkte sie ihm ein Käppchen von rotem Sammet, und weil ihm das so wohl stand und es nichts anders mehr tragen wollte, hieß es nur das Rotkäppchen ${ }^{26}$.

Il était une fois une douce fillette que tout le monde aimait rien qu'en la voyant, mais plus que tous sa grand-mère, qui ne savait que donner à l'enfant. Une fois, elle lui offrit un petit bonnet de velours rouge, et comme il lui allait si bien et qu'elle ne voulait plus porter autre chose, on ne l'appela plus que Petit-bonnet-rouge ${ }^{27}$.

$\mathrm{H}$. Pourrat reprend cette idée du «bonnet» en lieu et place de la bande de tissus hors de mode et, tout en faisant l'économie de l'adjectif "petit», il laisse entendre, dans son texte, le souvenir de Rotkäppchen, avec un "petit

25. Dictionnaire de l'Académie, édition de 1694, p. I68, disponible sur internet.

26. J. et W. Grimm, Kinder- und Hausmärchen, H. Rölleke (éd.), 200I, p. I56-160. Les citations suivantes renvoient aux mêmes pages de cette édition. Les traductions sont toujours les traductions de travail entreprises avec l'aide d'U. Heidmann et, sauf indication, c'est toujours moi qui souligne.

27. Traduction de travail par U. Heidmann et J.-M. Adam, au plus près de la langue, sans chercher l'élégance dans la langue d'arrivée. Ici aussi, sauf indication, c'est toujours moi qui souligne. 
bonnet d'écarlate», et du Petit Chaperon rouge, par la reprise, légèrement modifiée, du surnom :

Il y avait une fois une petite fille, qui allait sur ses huit ans et qui était toute gentille. Sa mère-grand lui disait toujours d'avoir grand-peur du loup, tant elle la trouvait gente : gente à donner envie de la croquer. Pour la rendre plus gente encore, la mère-grand lui fit faire un bonnet d'écarlate. De sorte qu'on ne nommait plus cette petite dans le pays que le Chaperon rouge ${ }^{28}$.

\section{Henri Pourrat : l'oralité d'une écriture}

Dans l'introduction au volume du Trésor des contes sous-titré Le bestiaire ${ }^{29}$, $\mathrm{H}$. Pourrat revendique d'être lu et compris comme un "conteur populaire». Il définit alors ses contes écrits par un principe de cohérence (ce qu'il appelle "redonner à chaque conte sa forme») et de "fidélité" à ce qu'il a recueilli. Dans une lettre à Alexandre Vialatte, du I3 décembre 1949, H. Pourrat définit très clairement son propos comme littéraire, mais il soumet l'esthétique (la forme stylistique et la composition) à l'éthique (authenticité et fidélité documentaire) : "J'ai fait ce qu'on n'avait encore jamais fait : le rassemblement sous forme littéraire mais exacte, sans fioritures ni fantaisies individuelles, des contes populaires ${ }^{30} »$. H. Pourrat se définit comme collecteur et comme transcripteur. «La première chose qu' ils exigent — dit-il des contes — c'est qu'on les retranscrive fidèlement", mais il ajoute immédiatement, dans le petit texte que B. Bricout place en ouverture du second volume du Trésor des contes: "C'est une bonne fortune d'en écrire un sous la dictée d'un conteur qui a le don. Mais quand ils vous arrivent tout aplatis et desséchés, il faut essayer de leur rendre vie et sève, en leur faisant retrouver leur fraîcheur à la source qui sourd dans l'herbe» (vol. II, p. virI).

H. Pourrat est aussi ambigu que les Grimm, comme le montre U. Heidmann dans sa contribution au présent volume. Il avait d'ailleurs recopié, dans un autographe retrouvé par B. Bricout, le fameux manifeste des frères Grimm :

Nous nous sommes fait une loi d'être fidèles et vrais. Nous n'avons rien ajouté de notre cru. Nous n’avons rien embelli. Nous avons donné le contenu de la légende comme nous l'avons reçu. Que l'expression, que l'exécution du détail vienne de nous

28. Le Trésor des contes, vol. I, p. 247. Par la suite toutes les références aux deux volumes de H. Pourrat seront indiquées par le numéro du volume, suivi de celui de la page. Sauf indication contraire, c'est moi qui souligne.

29. Note de H. Pourrat, en introduction du Trésor des contes. Le bestiaire, 1986, p. 15.

30. Note de Cl. Pourrat dans l'édition Folio du Trésor des contes. Les amours, I98I, p. 574. 
pour la plus grande part, bien entendu. Mais partout nous avons cherché à conserver la particularité qui nous avait frappés, afin que notre recueil offre de ce point de vue comme de tous les autres la vérité de la Nature même ${ }^{31}$.

Comme collecteur, H. Pourrat recueille un texte auprès d'un conteur auvergnat et, comme transcripteur, il le «restaure» en s'aidant d'éléments pris dans d'autres contes oraux ou imprimés. Son souci éthique de fidélité au récit entendu s'accompagne d'un travail d'écriture dont les manuscrits attestent l'ampleur ${ }^{32}$. Ce projet littéraire de passage de l'oral à l'oralité n'a pas l'ampleur de la réflexion sur le langage d'un Ramuz dont $\mathrm{H}$. Pourrat ne semble pas, selon J. Meizoz, comprendre les enjeux : «Pourrat commente [...] l'écriture ramuzienne comme si elle était une nature, une émanation immédiate de la "vie", à l'instar du parler des vignerons. Il semble souvent en négliger la mise en forme ${ }^{33}$." C'est, toujours selon J. Meizoz, la raison pour laquelle l'écrivain suisse romand s'est désolidarisé des écrivains régionalistes et qu'il a cherché à échapper à l'image restrictive que H. Pourrat lui-même donnait de son travail de la langue :

Au nom de la simplicité Pourrat pèche par simplification. [...] L'insistance de Pourrat à décrire Ramuz en "vigneron », en "Rousseau à l'état paysan», porte parole d'un petit peuple avec lequel il ferait corps témoigne, comme celle d'Henri Poulaille d'ailleurs, d'un certain aveuglement quant à l'identité sociale du Vaudois : Pourrat comme Poulaille dénient (ou masquent) l'universitaire lettré et citadin qu'était Ramuz ${ }^{34}$.

Malgré cette position de 1926, mon hypothèse est que H. Pourrat partage la réflexion générale de Ch. F. Ramuz sur l'oralité. D'ailleurs, la proximité de l'écriture de H. Pourrat et des thèses de Ch. F. Ramuz est telle que B. Bricout utilise, à plusieurs reprises ${ }^{35}$, la distinction introduite en 1926 par l'écrivain suisse entre la «langue-signe» et la «langue-geste»:

J'ai écrit (j'ai essayé d'écrire) une langue parlée : la langue parlée par ceux dont je suis né. J'ai essayé de me servir d'une langue-geste qui continuât à être celle dont on se servait autour de moi, non de la langue-signe qui était dans les livres. Et on me l'a vivement reproché ${ }^{36}$.

3I. Cité par B. Bricout, ouvr. cité, 2009, p. 178.

32. Voir, en particulier les travaux de D. Hadjadj (1988).

33. J. Meizoz, ouvr. cité, 200I, p. 9I.

34. Ibid. Les références entre guillemets portent sur un article de H. Pourrat : «Ramuz et le paysan", dans Pour ou contre C. F. Ramuz, un cahier de témoignages, Éditions du Siècle, Cahiers de la Quinzaine, 1926, p. I6I-162 et I64.

35. Dans son introduction du premier volume du Trésor des contes, 2009, p. XII et dans Le Savoir et la Saveur, 1992, p. 192-193.

36. Ch. F. Ramuz, «Lettre à Bernard Grasset», 1992 [1926], p. 53. J'en parle plus longuement dans Le style dans la langue, 1997, p. 59-63. 
Bien qu'aucune référence ne signale cet emprunt pourtant éclairant, B. Bricout cite deux prises de position de $\mathrm{H}$. Pourrat qui vont dans le sens de sa critique de la langue normée :

Du prospectus qui enveloppait la fiole de vermifuge au journal [...], de l'école à la radio, tout a enfoncé cette idée dans les têtes que pour être quelqu'un il faut user du morne et prétentieux jargon de la vie publique ${ }^{37}$ !

Et dans le sens également de sa pensée de l'oralité centrée sur le rythme :

Si tu ne refais pas du conte une nature, tu n'es pas transcripteur fidèle. Il faut que la mimique, les suspens, les changements de ton soient là, en dessous, de par une certaine figure du langage, de par le rythme; que le monde des champs et la vie des champs y soient aussi, sous l'écorce des choses, comme une pulpe d'expressions et d'images. Il faut que la forme, qu'une certaine saveur intérieure prêtent à la chose écrite le vivant goût de campagne de la chose parlée ${ }^{38}$.

Pour écrire Le Trésor des contes, H. Pourrat part de ce qu'il a entendu raconter, qu'il a noté sur de grandes feuilles de papier d'Ambert avec une vaste marge à gauche où interviennent, sur son texte de départ et jusqu'à obtenir la forme stabilisée, les opérations de remplacement, suppression, ajout, déplacement répertoriées par la génétique textuelle ${ }^{39}$. Il est intéressant de voir, comme le montre très bien B. Bricout, comment H. Pourrat retient des formules précises, notées sur ses carnets. Ainsi ce «belle qu'elle était fille / et de sa parure» dont nous retrouverons la trace dans une incise du Conte de Marie-Cendron. Une méta-réflexion attribuée par H. Pourrat à un maçon de Chassagnoles de 67 ans, Félix Bouche (entretien pris en note du 9 décembre 1978), résume superbement ce travail d'écriture :

Moi, j'étais maçon. Alors le manœuvre me faisait le mortier, n'est-ce pas? Alors le mortier, c'est moi qui vous le fais en ce moment et pour employer ce mortier, c'est-àdire les paroles, il faut en arranger un peu, vous comprenez. Parce que moi j’arrangeais mon mortier, je mettais de l'eau, je mettais un peu plus de ciment. Alors, ça c'est du rustique, ce que je vous dis. Alors, vous l'arrangerez à votre manière, vous y mettrez des phrases, des points d'interrogation, si vous voulez, des virgules et des apostrophes ${ }^{40}$.

37. H. Pourrat, Le blé de Noël, Marseille, Sagittaire, I942, p. 2II. Cité par B. Bricout, ouvr. cité, 1992, p. 192.

38. H. Pourrat, La porte du verger, Uzès, Éditions de la Cigale, I938, p. I44. Cité par B. Bricout, ouvr. cité, 1992, p. 193.

39. Voir à ce propos A. Grésillon : «Les manuscrits littéraires : le texte dans tous ses états», Pratiques, $\mathrm{n}^{\circ}$ 57, 1988 et J.-M. Adam : «Récritures et variation : pour une génétique linguistique et textuelle», Modèles linguistiques, XXX-I, vol. 9, 2009.

40. Cité par B. Bricout, ouvr. cité, I992, p. I74. 
Je ne considérerai pas ici l'opération génétique de récriture opérée par $\mathrm{H}$. Pourrat à partir des notes, mais les transformations effectuées dans une façon nouvelle de re-raconter quatre histoires déjà racontées par les Grimm et Perrault, et par ses informateurs. C'est dans la langue autant que dans la recomposition de l'intrigue que s'ancre cette autre façon de raconter. Cette autre manière réside dans ce qu'Henri Meschonnic théorise comme " oralité », qu'il oppose au parler et à l'oral et distingue de l'écrit :

[...] Concevoir l'oralité non plus comme l'absence d'écriture et le seul passage de la bouche à l'oreille, jadis infériorisé, aujourd'hui valorisé-psychanalysé par certains comme la pulsion libératrice [...]. Non, mais comme une organisation du discours régie par le rythme. La manifestation d'une gestuelle, d'une corporalité et d'une subjectivité dans le langage. Avec les moyens du parlé dans le parlé. Avec les moyens de l'écrit dans l'écrit. Et si quelque chose montre qu'il y a de l'oral dans l'écrit, et que l'oral n'est pas le parlé, c'est bien la littérature ${ }^{41}$.

Ce qui frappe d'entrée, c'est que l'univers des contes de H. Pourrat n'est que vaguement inscrit dans un terroir. Loin du modèle de la littérature régionaliste, la langue de ses contes n'est que teintée d'expressions vieillies et de quelques noms de lieux. Si un lexème paraît régional, il est généralement aussitôt clarifié en incise, comme dans ce passage du Conte de Marie-Cendron: "Ou c'était dans la souillarde des pots et des torchons qu'elle cherchait refuge, dans la buanderie, derrière le bugeadin, la grande jarre ronde à couler la lessive.» (vol. I, p. 54) C'est parfois par un nom de lieu comme le «bois de Malavieille» du Conte du Chaperon rouge que se marque une couleur locale. Une description comme celle du château où se retirent le roi et sa famille, un été, et qui deviendra le château de la Belle au bois dormant, dessine en réalité une géographie aussi floue et peu localisée que celle des contes des Grimm et de Perrault :

C'était en pays reculé, et la princesse n'y était jamais venue. Elle pouvait avoir quinze ans, alors. La voilà, encore une enfant, toute ravie du voyage, curieuse de ce pays nouveau, de la ramée qui remue aux deux bords du chemin, de l'eau qui tombe au flanc de la roche, de ces grands cierges de fleurs violettes montant d'une place de mousse, du vieux pivert à calotte d'écarlate qu'on voit voler de chêne en chêne. Au château, même chose : tant de degrés, de détours, de galeries, de corridors de pierre; et sortant de leur ombre au haut de quatre marches, d'une étroite fenêtre on voit tout par là-bas les pays bleus s'allonger et se perdre. (vol. I, p. 699)

La facture de cette description est très classique : motivation de la pause descriptive par la découverte du lieu par la jeune princesse, et point de vue porté à la fin par un «on voit» postiche. Bien loin de la littérature régiona-

4I. H. Meschonnic, La rime et la vie, 2006, p. 29I-292. 
liste, ces "pays bleus» dans le lointain ne sont pas aussi référentiellement situés que les paysages d'un Giono, d'un Daudet ou d'un Pagnol.

L'adjectif le plus fréquent pour faire allusion à la beauté et à la gentillesse de Cendrillon ou du Chaperon rouge est "gente», c'est-à-dire un mot vieilli. On trouve aussi de rares emplois d'adverbes vieillis : "On était déjà si loin qu'on ne voyait mêmement plus les toits des tours» (La Barbe-Bleue, vol. II, p. 277); sorte de mélange de même plus et sensiblement plus. Dans Le conte de la Belle au bois dormant, un certain nombre d'expressions vieillies ou constituées sur des modèles de langue anciens apparaissent, mais moins d'un par page : l'"ogrerie» (vol. I, p. 705) de la reine mère capable de dévorer «une pannerée de pommes» (ibid., p. 703) pour un plein panier de fruits, ou encore le "château en délabre» (ibid.) que découvre le jeune prince "venu courre la biche» (ibid., p. 70o) et «tout de jeune jeunesse» (ibid.). Aucun régionalisme vraiment, tout juste des termes techniques comme ce paysan «la houe à l'épaule, [qui] revenait de fossoyer son champ» (ibid., p. 7OI), au sens de creuser des fossés autour de son champ; ou encore des noms de plantes peu courantes pour les citadins, comme la «bourdaine», arbrisseau portant des baies noires. D'ailleurs ce paysan, comme la plupart des paysans de $\mathrm{H}$. Pourrat, ne parle absolument pas de façon rustique :

«Monsieur, dit l'homme, je le tiens de mon père, qui le tenait de son père, lequel le tenait de son grand-père à lui :

C'est par enchantement

La Belle au bois dormant!

La fille d'un roi, une jeune princesse y est endormie pour cent ans par quelque sort des fées. Un jour doit venir l'éveiller le fils aussi d'un roi. Adieu, monsieur, et je vous dirais bien de faire bonne chasse si vous ne pensiez pas que ce souhait coupât la chance.» (vol. I, p. 70I)

Mis à part le distique rimé, la langue de ce paysan est la même que celle de la voix narrative. La précision en apposition, «La fille d'un roi, une jeune princesse ", n'est qu'une très faible trace de reprise en ajout ou correction. La concordance des temps de la phrase périodique hypothétique finale est tout ce qu'il y a de plus orthodoxe. Toutefois, la comparaison avec ce que dit le «vieux Paysan» au Prince, dans le conte de Perrault, est éclairante :

Mon Prince, il y a plus de cinquante ans que j'ay ouï dire à mon pere, qu'il y avoit dans ce Chasteau une Princesse, la plus belle du monde; qu'elle y devoit dormir cent ans, \& qu'elle seroit reveillée par le fils d'un Roy, à qui elle estoit reservée. (I697, p. 2I)

Le discours direct du paysan de H. Pourrat est fait de phrases plus courtes que la longue phrase périodique de Perrault. Des répétitions produisent un rythme très différent de celui de la phrase de ce dernier, en particulier 
pour ce qui concerne la datation au rythme ternaire du début : «[...] je le tiens de mon père, / qui le tenait de son père, / lequel le tenait de son grand-père à lui $[\ldots]$. » Les relatives engendrent une progression thématique linéaire à effet marabout-bout de ficelle-selle de cheval-cheval de course ${ }^{42} \ldots$ La princesse de H. Pourrat n'est plus "la plus belle du monde», sœur de Psyché et rivale de Vénus dans les intertextes d'Apulée et de La Fontaine sur lequel avait travaillé Perrault ${ }^{43}$. Mais l'enchantement et le nom de la jeune princesse entremêlent la mémoire de la première partie du conte de Pourrat et de celui de Perrault. Nous pouvons être tentés de lire le souhait final du paysan au prince chasseur comme un appel à la chasse de la jeune princesse dont le destin sera — comme dans le conte de Perrault — d'être remplacée par une biche dans le festin terrible de sa future belle-mère ogresse.

Pour comprendre l'écriture de H. Pourrat, il est nécessaire de repartir de la "langue littéraire parlée» dont Charles Bruneau ${ }^{44}$ fait remonter la recherche aux Goncourt et Gilles Philippe au Daudet des Lettres de mon moulin, et surtout à Jules Vallès et à Jules Renard ${ }^{45}$. La tendance au style dépouillé, à la simplification du lexique et de la syntaxe, à la délittérarisation de la langue narrative, et pas seulement des personnages, était devenue, au tournant du XIX et du Xx ${ }^{\mathrm{e}}$ siècle, avec Anatole France, la norme littéraire. Dans un chapitre de La langue littéraire consacré à "Langue littéraire et langue parlée», G. Philippe souligne qu' "après la Seconde Guerre mondiale, on ne cherchera plus la langue parlée (paradigme oral), mais - selon la belle expression de Céline - "le souvenir de la langue parlée", ce qui est assurément autre chose ${ }^{46}{ }$. C'est ce que G. Philippe propose d'appeler le paradigme ou modèle vocal, celui que Ch. F. Ramuz a inventé, en compagnie du Giono de Colline (1929) et d'Un de Baumugnes (1929), et du Céline de Mort à crédit (1936). Le passage du modèle oral au modèle vocal tend à renouer avec la parole comme médium sonore. Selon moi, sans aller aussi loin, H. Pourrat va dans cette direction. L'écriture au long cours du Trésor des contes a été pour lui l'occasion d'un travail sur le «souvenir du conte dit et entendu» et d'une recherche d'un effet de vocalité ressemblant au contage oral.

La vocalité perce d'abord dans certaines formes d'interpellation de l'auditoire du conte. Ainsi, dans Le conte de Marie-Cendron : "La dame est morte,

42. Voir sur ce point les pages 75-80 de La linguistique textuelle (Adam, 20II).

43. Comme le montre U. Heidmann dans son article de Féeries $n^{\circ} 8:$ «Expérimentation générique et dialogisme intertextuel : Perrault, La Fontaine, Apulée, Straparola, Basile», 20II, p. 45-69.

44. Ch. Bruneau, Histoire de la langue française, XII-2, Paris, Armand Colin, 1972, p. I09.

45. G. Philippe, La langue littéraire, 2009, p. 64-65.

46. Ibid., p. 84 . 
bonnes gens...» (vol. I, p. 53); «Pensez donc, quand on a pour marraine une fée...» (vol. I, p. 57); «Mais elle avait seize ans, ou bien mettez dix-sept. Est-on sur ses gardes, à cet âge?» (vol. I, p. 57); ou encore : «La mère de ces filles s'en mêla, la marâtre de la Cendroune. Vous le savez, elle était un peu sorcière. " (vol. I, p. 59). Il en va de même dans Le conte de la Barbe-Bleue, d'abord lorsqu'il est question de sa fortune: «Imaginez ce qu'il avait amassé de ses six femmes!» (vol. II, p. 274); ou dans cette parenthèse en forme d'arrêt sur mot du narrateur, lorsque l'époux amène sa femme au bord de l'étang où il compte bien l'exécuter : "(Savez-vous ce que c'est qu'une île? Moi, je ne le sais pas, je n'en ai jamais vu.)» (vol. II, p. 277); le dénouement comporte encore une de ces interpellations de l'auditoire : «Enfin, devant la porte du château, elle rencontra ses frères qui arrivaient au triple galop, fondus de sueur. Vous pensez quelles embrassades.» (vol. II, p. 278).

Hors des dialogues, les exclamatives sont très nombreuses. Par exemple dans Le conte de Marie-Cendron, à propos des deux méchantes sœurs : "Les deux autres, en devenant des personnes, quel mépris pour Marie!» (vol. I, p. 54); lorsque le fils du roi croise les yeux de Marie-Cendron les suspensions de la phrase se mêlent aux exclamations : "Comme les yeux, des fois, se parlent et s'engagent : elle aussi, du premier regard... Mon Dieu, ce soleil tout d'un coup à en perdre la tête! Alors, mon Dieu, mon Dieu, elle avait tant de prières à faire pour s'y reconnaître! Tant à demander au bon Dieu...» (vol. I, p. 57) ; ou quand les deux sœurs se moquent de MarieCendron, la voix narrative s'emporte par empathie : «Quelles risées, quelles dérisions des deux pecques!» (vol. I, p. 58); l'épisode de l'essayage de la pantoufle de verre n'est pas en reste: "Des filles du pays, comme chacune désirait que son pied pût chausser la pantoufle de verre!» (vol. I, p. 59), et lorsque le roi est obligé de partir avec la sœur cadette qui vient de passer la pantoufle : «Il avait donné sa parole, le malheureux!...» (vol. I, p. 59)

Un autre trait stylistique marquant de l'écriture de $\mathrm{H}$. Pourrat est certainement l'usage de "voilà» qui se retrouve dans tout le corpus et n'est pas loin des «da» et "nun» des Grimm, que décrit ici-même Cyrille François et qui embarrassent tant les traducteurs qu'ils les suppriment généralement. Dans Le conte de Marie-Cendron, de nombreux "voilà» suivent et ponctuent les moments importants de l'intrigue : "Voilà comment Marie sut se mettre à distance» (vol. I, p. 54); "La voilà à ce ramassage. Mais les pois roulaient dans les coins, sous le fagot [...]" (vol. I, p. 55); "Voilà Marie-Cendron changée en grande dame» (vol. I, p. 56); «Voilà le fils du roi seul avec sa pantoufle» (vol. I, p. 58); "Voilà les deux sœurs, les dames, les demoiselles à s'entreregarder, à murmurer, à lamenter.» (vol. I, p. 60). Dans Le conte de la Belle au bois dormant, bien que moins nombreux, ils participent aux effets 
d'hypotypose : «Or, voilà que le jour du baptême [...]» (vol. I, p. 697); «Elle pouvait avoir quinze ans, alors. La voilà, encore une enfant, ravie du voyage" (vol. I, p. 699); "Voilà qu'elle s'éveille» (vol. I, p. 702). Le conte de la Barbe-bleue n'en présente qu'une occurrence au début de l'histoire : "Voilà qu'ils se sont mariés» (vol. II, p. 274). De même pour Le conte du Chaperon rouge, cette fois dans l'épisode ajouté du repas cannibale de la petite fille : "Voilà le Chaperon rouge s'affairant et soupant» (vol. I, p. 249).

La langue littéraire de $\mathrm{H}$. Pourrat s'approche encore du modèle vocal lorsque la parole de la voix narrative mime la simplicité directe de l'oral, ou introduit des expressions orales. Ainsi, dans Le conte du Chaperon rouge, lorsque le loup découvre la mère-grand endormie et que la voix narrative introduit le refrain d'une berceuse bien connue : «[...] elle s'était tout de suite fourrée au lit et dormait là comme Colas mon petit frère» (vol. I, p. 248), et plus encore dans cette phrase : «Et hop, d'un seul coup de gueule, le loup la gobe comme le loriot gobe la cerise» (vol. I, p. 250). On a reconnu là l'épisode tragique de la dévoration de la petite fille, qui s'ouvre par une interjection et se termine par une comparaison concrète, ancrée dans l'environnement naturel paysan.

Ce type de comparaison naturalisante, qui correspond au projet de $\mathrm{H}$. Pourrat et prolonge l'entreprise des Grimm, se retrouve un peu partout dans les contes. Ainsi dans le même Conte du Chaperon rouge, lorsque le loup vient de dévorer la mère-grand : "[...] l'air content comme un chat qui vient de lécher le beurre» (vol. I, p. 248); lorsque la petite fille arrive chez sa mère-grand : "Entre le Chaperon rouge, sautant comme un perdreau» (vol. I, p. 249); enfin lorsque surgit le parrain bûcheron : "Effaré comme la lune rousse, le loup s'était dressé en pied» (vol. I, p. 250). Il en va de même dans cette phrase nominale du Conte de Marie-Cendron: "Sa robe, brillante comme un semis d'étoiles!» (vol. I, p. 56) Plus encore dans Le conte de la Barbe-Bleue: "[...] on l'appelait la Barbe-Bleue. C'était à cause d'une barbe qu'il avait noire comme l'aile $d u$ corbeau, si fort noire qu'elle en était bleue" (vol. II, p. 274) et, peu après, à propos de celle qui décide de l'épouser : "Mais elle, elle n'avait pas peur de cette barbe. Vive comme un poisson, fine comme une abeille, et prête à se jeter au travers de tous les hasards» (vol. II, p. 274); quand la jeune épouse découvre les richesses de Barbe-Bleue : "des dentelles comme des toiles d'aragne» (vol. II, p. 275); ou plus loin : «La Barbe-Bleue l'emmenait d'un tel train qu'ils volaient comme la tempête et comme un orage de mer» (vol. II, p. 277). Ou encore dans Le conte de la Belle au bois dormant, quand la jeune princesse «s'émerveille de voir le fuseau virer, en grossissant, sous le fil qui naît de lui, virer, monter, descendre, comme un moucheron, comme un bourdon qui danserait dans 
l'air» (vol. I, p. 699); lorsque le prince s'avance en direction de la belle endormie : "Â un des arbres, il attacha le cheval et continua de son pied léger, comme un garçon qui va aux noisettes» (vol. I, p. 702); "Le cuisinier s'est retiré, tremblant comme la feuille» (vol. I, p. 704); «Un beau matin, toute reprise d'appétit, elle est venue chez son cuisinier. Comme le chat qui part à la chasse aux souris, elle allait le long des murailles, guettant les marmots de par là» (vol. I, p. 705), et lorsque la reine mère ogresse découvre que son cuisinier l'a trompée : «Elle les apostrophe de tout près, comme une chienne qui veut mordre» (vol. I, p. 706).

Une même dynamisation vocale se retrouve à la fin du Conte de la Barbe-bleue : "Cela fait, ils sont revenus chez la Barbe-Bleue. Et allez! Ils ont mis le feu partout» (vol. II, p. 278). La simple insertion de «allez!» dans l'énoncé consécutif : «Et [...] ils ont mis le feu partout» transforme la phrase écrite standard en oralité montrée. Il en va de même dans la deuxième phrase de ce paragraphe de la fin du conte où la voix narrative partage la terreur du personnage :

Elle ouvre la porte, à la fin des fins. Malheur de mon cœur! Quand elle voit ces murs, ce plancher plein de sang, les cheveux lui lèvent sur la tête. Une telle frayeur lui vient qu'elle se met à trembler de tous ses membres. Et ne lâche-t-elle pas cette clef? (vol. I, p. 275)

La dernière phrase est une forme particulière d'hypotypose : la narration suit à ce point l'action que la voix narrative s'étonne et s'interroge tout en décrivant le fait que la jeune épouse lâche la clé dans le sang. Cette tension peut devenir telle que le discours narratif touche au discours indirect libre, comme dans la scène de l'essayage des pantoufles de verre de Marie-Cendron :

On passe à la cadette. Misère et peste! Elle n'entrait pas trop non plus... Mais quoi? toutes les filles du pays, déjà, avaient manqué l'essai. Il en fallait pourtant bien une! La mère de ces filles s'en mêla [...]. (vol. I, p. 59)

Des sortes d'énoncés évaluatifs peuvent ponctuer la narration. Ainsi quand les yeux du prince croisent ceux de la belle endormie qui s'éveille, après avoir dit qu'entre eux «l'entente s'est faite d'un grand regard", un énoncé répété sert de transition intensive et émotive : "C'est le sort, c’est le sort. Autour d'eux, dans le château, tout le monde s'éveille» (vol. I, p. 702). Ou encore, dans le même Conte de la Belle au bois dormant, quand le roi part à la guerre: "Mais s'est élevée une grande guerre. Ces rois! Toujours en guerre! Comme disait l'autre, il faudrait les faire boire et les rapatrier, avant quils en arrivent à ces grosses sottises» (vol. I, p. 704). Tout naturellement, la tension dramatique de la fin du conte - sur le modèle de celui de Perrault, mais sans son ironie — bouscule la syntaxe (interjection, phrase exclamative, infranomination (“ç’a»), phrases nominales : 
Ah, tout à coup, ça été le feu de Dieu! Une poussière en tourbillon s'élève. Le roi, le jeune roi! Il revient de la guerre. Bride abattue, tant il lui tarde de retrouver les siens. Il est bien inspiré d'arriver comme la foudre. (vol. I, p. 706)

Plusieurs opérations stylistiques propres à $\mathrm{H}$. Pourrat se concentrent dans une réplique du Chaperon rouge : «- $O h$, mère-grand, comme vous avez bourrues vos pauvres jambes, plus bourrues que les sapins du bois de Malavieille» (vol. I, p. 249) Cette réplique vient d'une forme beaucoup plus simple de Perrault : «[...] ma mere-grand que vous avez de grandes jambes? c'est pour mieux courir mon enfant» (I697, p. 55); cette question sur les jambes est absente, en revanche, du texte des Grimm. Par contraste, le texte de H. Pourrat apparaît comme travaillé par une récriture qui le concrétise, lui confère une émotivité accrue et une poéticité par un usage des répétitions portées au rang d'unité de rythme. L'attaque par l'interjection "Oh" marque la surprise et n'ajoute à l'énoncé qu'une plus grande force émotive. La répétition de "bourrues", dans un emploi très concret, souligne le caractère non dégrossi des jambes de l'aïeule, en privilégiant ici encore la comparaison naturalisante. Cette comparaison est non seulement ancrée dans l'environnement naturel du bois de sapin, mais ce bois est, dans un second temps perceptif, localisé dans un toponyme régional supposé connu des auditeurs/lecteurs, et bien choisi pour thématiser toutes les souffrances de la vieillesse : Mal-à-vieille. Enfin le déplacement de l'épithète «bourrues» à gauche du syntagme nominal : "comme vous avez bourrues vos pauvres jambes » brise la linéarité de la phrase canonique en emphatisant la propriété. Le travail du style, qui poétise indéniablement le texte, est également lisible dans la réplique du loup :

\author{
C’EST DE vieillESSE [4 syllabes], \\ ma petite fille [ 5 syllabes], \\ C'EST DE traîtrESSE : [4 syllabes] \\ j'ai tant couru les BOIS [6 syllabes] \\ que je suis devenue BOIS! [7 syllabes]
}

L'intensive consécutive "tant... que...» articule un énoncé coupé à la sixième syllabe, sur le mot "bois» qui fait office de rime intérieure.

À côté de ce genre d'énoncé, on trouve aussi bien des phrases complexes au passé simple, à fort développement à gauche du verbe, et à la diction seulement hachée par une surponctuation de virgules, comme dans ce début du Conte de la Barbe-Bleue: "La surveille des noces, pourtant, la fille, qu'il avait demandée pour être la septième, vit ses frères venir lui parler dans sa chambre.» (vol. II, p. 274) 
La présence du discours indirect libre vient souvent contaminer le discours narratif et lui donner une puissante force d'expressivité et d'oralité qui accentue la dramatisation et la participation émotionnelle de la voix du conteur aux événements vécus par les personnages. Ainsi dans ce passage du Conte de la Barbe-Bleue: "Sitôt la lettre reçue, ses frères avaient sauté à cheval et ils étaient partis ventre à terre; mais c'était si loin! c'était si loin! Ils ne pouvaient être là avant le soir.» (vol. II, p. 276)

Le discours direct libre est également fréquent, réduisant, dans Le conte $d u$ Chaperon rouge, la frontière entre le discours narratif et la parole des personnages : "[...] et là la mère-grand demanda à la petite par quel chemin elle voulait retourner : celui des épinettes ou celui des pierrettes?» (vol. I, p. 247)

Il n'est pas toujours besoin du discours indirect libre pour oraliser le discours du narrateur. Ainsi dans ce passage du Conte de Marie-Cendron qui commence par les phrases courtes (PI, P2, P6) de l'écriture délittérarisée dont nous parlions plus haut et se poursuit par une oralisation et une vocalisation :

[PI] La marraine fit un autre pas. [ $\left.\mathrm{P}_{2}\right]$ De sa baguette, elle toucha Marie. $\left[\mathrm{P}_{3}\right]$ Voilà Marie-Cendron changée en grande dame. [ $\left.\mathrm{P}_{4}\right]$ Si belle, — belle qu'elle était fille, et belle de sa parure, - qu'il n'y avait pas princesse! [ $\left.\mathrm{P}_{5}^{1}\right]$ Sa robe, brillante comme un semis d'étoiles! $\left[\mathrm{P}_{5}^{2}\right]$ et pour pantoufles, des pantoufles de verre!

[P6] Ainsi mise, la fée l'envoya à l'église. [P7] Mais ce fut en lui recommandant de sortir avant le dernier évangile. [P8] — Peut-être que l'habillement était fée? Peutêtre que l'enchantement ne devait tenir que juste pour ce temps de la messe? [P9] Toujours est-il que si la Cendroune n'était pas sortie au juste moment, il lui arriverait malencontre. (vol. I, p. 56)

$\mathrm{P}_{3}$ est un très bon exemple des phrases commençant par «Voilà» qui font étymologiquement voir là le personnage, dans un énoncé nominal qui supprime le processus au profit de l'état nouveau du personnage. Dès $\mathrm{P}_{4}$ et la suite de phrases nominales qui constitue $\mathrm{P}_{5}$, les points d'exclamation et les tirets se chargent de déconstruire la phrase en l'affectivisant et en l'oralisant. La corrélative intensive syntaxiquement puissante est interrompue entre la protase et l'apodose : «SI belle, — [...], - QU'il n'y avait pas de princesse!» L'intensif caractéristique de la fictionnalité du conte ${ }^{47}$ devient ici marque d'oralité. L'incise «belle qu'elle était fille, et belle de sa parure» est comme une correction-précision apportée à l'épithète marquée par l'intensif : il s'agit de redoubler la beauté d'origine physique par la beauté extérieure du costume; ce qui permet le superlatif «qu'il n'y avait pas

47. J.-M. Adam, ouvr. cité, 2010, p. 249-262. 
princesse ». Ce mécanisme d'interruption caractéristique de l'oralité est ici intégré à un parallélisme proche du décasyllabe $(5+\mathrm{ET}+5$ syllabes $)$. Sur cette lancée, $\mathrm{P}_{5}$ est dominé par les phrases nominales exclamatives présentant une prédication intéressante : la perception d'abord de la robe et des pantoufles (thèmes), et une prédication virant à la comparaison ( $\left.\mathrm{P}_{52}\right)$, puis en reformulation du thème et adjonction d'un complément de nom, comme si la perception de la matière surprenante des pantoufles survenait dans un second temps. L'intensification émotive lancée par l'intensive consécutive déborde et en vient à empêcher la prédication verbale. L'implication évaluative du narrateur rejoint ainsi celle des personnages spectateurs du conte.

Les interrogations de P8 sont intéressantes car elles ne peuvent être considérées comme la forme d'assertion déguisée des questions rhétoriques. Il s'agit bien d'une suspension (certes feinte) du pouvoir omniscient de la voix narrative. Le "Toujours est-il que» n’apporte pas de réponse aux questions posées et fonde l'enchaînement narratif sur une forme tronquée de concessive alternative de type: Qu'un certain état de choses soit ou ne soit pas, cela ne change rien à la vérité de ce qui suit. En d'autres termes, "Toujours est-il que» laisse entendre que ce qui suit est narrativement seul digne d'intérêt et que, rétroactivement, l'état de choses auquel la locution renvoie doit être considéré comme une question secondaire. C'est là qu'intervient la phrase $\left(\mathrm{P}_{9}\right)$ : «Toujours est-il que si la Cendroune n'était pas sortie au juste moment, il lui arriverait malencontre.» Cet énoncé, très proche d'un discours rapporté, donne à entendre, dans les mots du narrateur, les paroles de la fée et, au-delà, celles de la fée marraine du conte de Perrault :

Mais sa Maraine luy recommanda sur toutes choses de ne pas passer minuit, l'avertissant que si elle demeuroit au Bal un moment davantage, son carosse redeviendroit citroüille, ses chevaux des souris, ses laquais des lezards, \& que ses vieux habits reprendroient leur premiere forme. (I697, p. I3I)

La phrase P9 présente par ailleurs un usage de l'épithète "juste» en lieu et place de la collocation «bon moment» attendue, et un mot vieilli : "malencontre» qui joue ici avec son sens établi de mauvaise rencontre en y ajoutant, comme dans un mot-valise, le malheur présent dans la collocation arriver malheur, ici transformée.

Pour baliser l'oralité de cette écriture, il faudrait encore dire un mot des énoncés formulaires qu'étudie fort bien B. Bricout ${ }^{48}$. Dans Le conte de la Barbe-Bleue, quatre petits distiques exclamatifs ponctuent l'épisode le

48. B. Bricout, ouvr. cité, I992, p. 245-259. 
plus dramatique du récit. D’abord le dernier verre de la condamnée que son terrible époux se prépare à exécuter comme ses précédentes femmes :

Buvez, buvez la belle,

Buvez de ce vin blanc! (vol. II, p. 277)

Ensuite la ruse qui va inciter Barbe-Bleue à se retourner alors qu'il est au bord d'un étang et ne sait manifestement pas nager :

Ce n'est pas l'honneur d'un chevalier

De voir sa femme déshabillée! (vol. II, p. 277)

Un troisième distique renforce la réponse implacable de celle qui refuse de secourir l'ennemi qu'elle vient de précipiter dans l'étang où il est en train de se noyer :

Ce n'est pas moi, maudit larron,

Mais les poissons te mangeront! (vol. II, p. 278)

Enfin un dernier distique marque le terme de cet épisode profondément différent des récits de Perrault et des Grimm :

Le cheval gris m'a emmenée bien tristement,

Le cheval blanc me ramènera bien joyeusement! (vol. II, p. 278)

Le conte de la Belle au bois dormant reprend par deux fois le petit distique exclamatif déjà cité. Situé à la transition des deux parties du conte, il apparaît d'abord dans la bouche du paysan qui révèle au prince l'existence du château et de la belle endormie; on le retrouve ensuite dans la bouche des serviteurs qui s'éveillent de leur sommeil séculaire :

C'est par enchantement

La Belle au bois dormant! (vol. I, p. 70I-702)

Ce conte est un des rares à comporter une morale et à se terminer par trois vers en forme de comptine de clôture :

Et le coq de l'aurore chanta,

Le coq du jour claironna,

Et le conte finit là. (vol. I, p. 707)

Le conte du Chaperon rouge reprend aussi par deux fois la formulette célèbre de Perrault "tire la chevillette, la bobinette cherra», en la revisitant rythmiquement pour en faire un distique de deux fois six syllabes :

- Tire la chevillette

Cherra la bobinette! (vol. I, p. 248-249) 
Par un quatrain de vers de quatre syllabes, le chat avertit en miaulant de son cannibalisme un Chaperon rouge affamé (modification majeure des contes de Perrault et des Grimm qui n’aura toutefois pas d'effet néfaste sur l'intégrité corporelle de la mère-grand ressuscitée) :

Tu manges la chair

De ta grand-mère,

Tu bois le sang

De ta mère-grand! (vol. I, p. 249)

De la même façon, Le conte de Marie-Cendron est ponctué par les redoublements de distiques qui alternent et varient légèrement le texte. C'est d'abord l'intervention de la fée qui vient au secours de la pauvre Marie-Cendron, empêchée de se rendre à la messe par l'épreuve des petits pois dispersés sur le sol par sa sadique marâtre (comme dans le conte des Grimm) :

Par la vertu de ma baguette

Que les pois rentrent en la bogette! (vol. I, p. 55)

C'est ensuite la réplique chuchotée de Marie-Cendron à ses sœurs qui n'ont pas identifié la véritable identité de la belle princesse apparue à la messe et enfuie au dernier évangile :

- Pour gente et gente qu'elle soit,

Toujours pas plus gente que moi! (vol. I, p. 56)

La fée vient de nouveau au secours de la pauvre Marie-Cendron empêchée de se rendre à la messe par une nouvelle épreuve imposée par sa marâtre :

Par la vertu de ma baguette

Toutes les cendres en leur logette! (vol. I, p. 57)

Survient une nouvelle réplique chuchotée de Marie-Cendron à ses sœurs toujours aussi mystifiées par elle :

Si gente et gente qu'elle soit,

Toujours pas plus gente que moi! (vol. I, p. 58)

Ces distiques rappellent ceux du conte des Grimm. D'abord lorsque, par deux fois, les colombes, tourterelles et oiseaux du ciel viennent en aide à Aschenputtel :

Die guten ins Töpfchen,

die schlechten ins Kröpfchen. (1857, p. 139)

Les bonnes dans le petit pot,

Les mauvaises dans le jabot. 
C'est ensuite, par trois fois, l'appel à l'aide au petit noisetier magique :

Bäumchen, rüttel dich und schüttel dich,

wirf Gold und Silber über mich. (1857, p. 14I)

Petit arbre, agite-toi et secoue-toi, jette de l'or et de l'argent sur moi.

Chez H. Pourrat enfin, le prince entend un oiselet par deux fois « ramager sa chanson" pour l'avertir du fait qu'il est trompé et que les deux sœurs n'ont - comme dans le conte des Grimm — chaussé la pantoufle que par supercherie :

Tu prends la méchante

Tu laisses la gente

Toute seulette en son coin

Derrière le bugeadin. (vol. I, p. 59)

Tu prends la méchante

Tu laisses la gente

Toute seulette en son coin

Derrière le bugeadin.

Tu prends la méchante

Tu laisses la gente (vol. I, p. 6o)

Ces deux strophes reprennent les deux premières des trois strophes suivantes du conte des Grimm (dont nous proposons une traduction de travail). À deux occasions, lors du passage des deux sœurs usurpatrices, deux petites colombes sur le noisetier magique s'écrient :

Rucke di guck, rucke di guck,

Blut ist im Schuck:

der Schuck ist zu klein,

die rechte Braut sitzt noch daheim. (1857, p. I43)

Roucouregarde, roucouregarde,

du sang dans la pantoufle :

la pantoufle est trop petite,

la vraie fiancée est encore là où elle habite.

Après le triomphe d'Ascheputtel, elles corrigent leur dire :

Rucke di guck, rucke di guck,

kein Blut im Schuck:

der Schuck ist nicht zu klein,

die rechte Braut, die führt er heim. (1857, p. I44)

Roucouregarde, roucouregarde, pas de sang dans la pantoufle : 
la pantoufle n'est pas trop petite,

c'est la vraie fiancée qu'il mène où il habite.

Manifestement, le conte de H. Pourrat reprend les formulettes rimées du conte des Grimm dont il modifie à peine la cruauté du dénouement : yeux crevés des méchantes sœur chez les Grimm et mort plus radicale encore chez H. Pourrat :

Il n'y eut que les deux sœurs et la marâtre... Celles-là, leur dépit leur donna la jaunisse. Elles en trépassèrent au bout de la semaine. Marie-Cendron les a pleurées un peu; mais avant l'autre semaine, le fils du roi, son cher et tendre, l'avait bien consolée. (vol. I, p. 6I)

\section{Le conte de la Belle au bois dormant de Pourrat est-il une «simple amplification » de celui de Perrault?}

Ceci nous amène au dernier point que je voudrais succinctement traiter : les différences assez profondes des intrigues. Je ne reviens pas sur le fait que H. Pourrat opte pour le conte en deux parties de Perrault contre l'histoire simplifiée des Grimm qui, en supprimant la seconde partie, évitent la mise en évidence des dangers encourus par les époux princiers soumis, comme Psyché, aux persécutions d'une belle-mère ogresse digne de Vénus.

Alors que les Grimm font s'endormir non seulement tout le château, mais le roi et la reine, afin que soit reconstitué, un siècle plus tard, la famille initiale, selon une des règles qui préside à l'idéologie des "contes du foyer", H. Pourrat adopte le même schéma que Perrault en le dramatisant un peu et en ajoutant un ministre :

Le roi n'a eu que le temps de se coiffer de sa couronne de roi, la reine de sa couronne de reine, le ministre de mettre la main sur ses papiers de ministre - il fallait bien, puisqu'ils avaient le royaume en charge - et ils ont laissé là la Belle au bois dormant. (vol. I, p. 700)

Le jugement de valeur de G. Rouger, dont nous parlions plus haut : "médiocre amplification du récit de Perrault», est tout à fait contestable. H. Pourrat supprime par exemple tout l'épisode de la venue de la jeune fée au moment de l'endormissement de la princesse. Cette suppression est assez conséquente pour compenser quelques expansions d'épisodes, comme par exemple celui de la proclamation de l'édit royal. Chez Perrault, comme chez les Grimm, une phrase suffit :

Le Roi pour tâcher d'éviter le malheur annoncé par la vieille, fit publier aussitost un Edit, par lequel il deffendoit à toutes personnes de filer au fuseau, ny d'avoir des fuseaux chez soy sur peine de la vie. (Perrault, I697, p. 8-9) 
Der König, der sein liebes Kind vor dem Unglück gern bewahren wollte, liess den Befehl ausgehen, dass alle Splindeln im ganzen Königreiche sollten verbrannt werden. (Grimm, I857, p. 258)

Le roi, qui voulait préserver son cher enfant du malheur, donna l'ordre de brûler tous les fuseaux du royaume entier.

Chez H. Pourrat, en revanche, l'épisode est profondément dramatisé, phrases nominales, exclamatives sont accumulées, le présent narratif remplace le passé simple, les discours direct libre et indirect libre surgissent :

Le roi ne balança pas. Sur-le-champ, il manda quatre cents de ses trompettes. Par tout le royaume des Alouettes, commandement de brûler tous les fuseaux! Plus de fuseau, même au fond des maisons, dans le recoin du coffre ou sur la tablette de la cheminée, plus un seul! L'édit bien trompetté, dans les villes, villages, châteaux et métairies, il envoie des gens tout partout : ils doivent s'assurer que tous les fuseaux ont disparu. Mais comment filera-t-on? Se passer de fil, on ne le peut : il faut coudre et tisser! Les personnes ont besoin de toiles et de serges.

Le roi n'a rien voulu savoir. La première chose, c'est qu'il n'y ait pas de fuseau pour blesser la princesse. (vol. I, p. 698)

C'est alors que $\mathrm{H}$. Pourrat intègre dans la fiction l'évolution technique et l'invention historique du rouet :

De fait, nécessité rend inventif. Les artisans et les savants ont tous fait travailler leur tête. Un beau soir, à la reine, on a apporté un rouet. Les femmes se sont mises à filer au rouet : bien plus uniment, bien plus vite! Bientôt il n'y a plus eu que rouets dans le royaume. La princesse a grandi sans jamais voir de fuseau. (vol. I, p. 698-699)

Tout l'humour et l'écriture de H. Pourrat ne paraissent concentrés que dans cette seule transformation. Nous avons déjà vu plus haut les autres traits de cette écriture.

\section{Débuts et fins des «Chaperons rouges»}

À l'inverse du conte précédent, H. Pourrat suit plutôt ici les Grimm. Au cœur du conte de Perrault, le narrateur souligne la naïveté de la "petite fille de Village» par une relative chargée d'expliciter un présupposé qui a la forme d'une assertion impersonnelle au présent de vérité générale : «La pauvre enfant, qui ne sçavoit pas qu'il est dangereux de s'arrester à écouter un Loup, luy dit [...].» (1697, p. 49) Cette relative prédicative en apposition met en évidence un danger connu de tous, mais dissimulé par la mère et la grand-mère qui omettent de mettre la jeune fille en garde contre les rencontres de «loups doucereux»(comme les définit la moralité). 
Les contes des Grimm et de Pourrat corrigent ce défaut des mères et la mise en accusation, par Perrault, qui en découle ${ }^{49}$. Un jeu avec la langue nous ramène à l'expression du début du conte de Perrault : «Il estoit une fois une petite fille de Village, la plus jolie qu'on eut sçu voir.» Alors que l'adjectif «belle» est généralement utilisé pour décrire les héroïnes, le choix de «jolie» doit être revu à la lumière du Dictionnaire de l'Académie qui précise que cet adjectif signifie «Gentil, agreable. Il ne se dit guere que de ce qui est petit en son espece, \& qui plaist plustost par la gentillesse que par la beautéso ». Cet énoncé est surtout très proche d'une forme déjà lexicalisée au XVII ${ }^{\mathrm{e}}$ siècle, d'après le Dictionnaire de l'Académie : "On dit d'un enfant joly et beau, qu'Il est joly à manger, qu'il est à manger." Le destin de la petite fille est ainsi fixé dans la langue. La mère et la grand-mère sont «folles» de jeter leur Petit Chaperon rouge "dans la gueule du loup ${ }^{51}$ ".

Cette façon détournée de dire les choses en jouant avec la langue apparaît mieux quand on compare l'ouverture du conte de Perrault avec celle du Conte $d u$ Chaperon rouge dans lequel $\mathrm{H}$. Pourrat combine manifestement des éléments des textes de Perrault et des Grimm : "Il y avait une fois une petite fille, qui allait sur ses huit ans et qui était toute gentille. Sa mèregrand lui disait toujours d'avoir grand-peur du loup, tant elle la trouvait gente : gente à donner envie de la croquer.» (vol. I, p. 247) Dans ces lignes, la mère-grand, qui élève seule sa petite fille, énonce clairement le danger et le double sens en langue de "gente à croquer» est rendu manifeste par la voix narrative. On mesure ainsi tout ce qui sépare un récit explicite d'un récit fondé, comme celui de Perrault, sur l'équivoque et le jeu polysémique, fait d'humour et d'ironie.

Chez les Grimm, la petite fille n'est plus «la plus jolie qu'on eut sçu voir», et la connotation de "jolie à croquer» disparaît. La mise en garde maternelle corrige l'inconséquence dénoncée par le conte de Perrault, en centrant l'interdit sur le fait de ne pas s'écarter du droit chemin :

Eines Tages sprach seine Mutter zu ihm : "Komm, Rotkäppchen, da hast du ein Stück Kuchen und eine Flasche Wein, bring das der Großmutter hinaus; sie ist krank und schwach und wird sich daran laben. Mach dich auf, bevor es heiß wird, und wenn du hinauskommst, so geh hübsch sittsam und lauf nicht vom Weg ab, sonst fällst du und zerbrichst das Glas, und die Großmutter hat nichts. Und wenn du in ihre Stube kommst, so vergiß nicht, guten Morgen zu sagen, und guck nicht erst in alle Ecken herum."

49. Analysée par U. Heidmann, ouvr. cité, 20IO, p. I07-III.

50. Dictionnaire de l'Académie, 1694, p. 666.

51. Comme le dit également le Dictionnaire de l'Académie, I694, p. 666. 
"Ich will schon alles gut machen", sagte Rotkäppchen zur Mutter und gab ihr die Hand darauf. (1857, p. I57)

Un jour sa mère lui parla ainsi : "Viens, Petit-bonnet-rouge, voici un morceau de gâteau et une bouteille de vin, porte ceci à ta grand-mère; elle est malade et faible et elle va bien s'en régaler. Mets-toi en route, avant qu'il ne fasse trop chaud, et dès que tu sors, sois bien sage et ne t'écarte pas du chemin, autrement tu tombes et tu casses le verre, et la grand-mère n'a rien. Et quand tu entres dans sa chambre, n'oublie pas de dire bonjour, et ne regarde pas d'abord dans tous les coins.»

"Je veux bien faire tout bien", dit Petit-bonnet-rouge à sa mère et elle lui tapa dans la main en guise de promesse.

Contrastivement, $\mathrm{H}$. Pourrat introduit la mise en garde dès les premiers mots et il modifie la configuration familiale en étant le seul à faire vivre la petite fille chez sa grand-mère. Mais sa famille est agrandie par le parrain bûcheron qui la délivre, à la fin du conte. Il remplace la mère inconséquente par un bûcheron venu du conte des Grimm, et déjà présent dans l'arrière-plan du conte de Perrault : «[...] le Loup [...] eut bien envie de la manger, mais il n'osa, à cause de quelques Bucherons qui estoient dans la Forest.» (I697, p. 49)

C'est surtout par leur dénouement que les trois contes diffèrent. Alors que celui de Perrault s'arrête à la dévoration de la jeune fille par le loup, autant Pourrat que les Grimm évitent le conte d'effroi en proposant une fin heureuse qui le transforme en conte d'avertissement. Une fois libérées par un chasseur qui d'un coup de hache délivre la grand-mère et l'enfant, une fois les pierres mises dans le ventre du loup qui s'effondre et tombe raide mort, Petit-bonnet-rouge tire elle-même, en pensée, la morale de l'histoire :

Da waren alle drei vergnügt; der Jäger zog dem Wolf den Pelz ab und ging damit heim, die Großmutter aß den Kuchen und trank den Wein, den Rotkäppchen gebracht hatte, und erholte sich wieder, Rotkäppchen aber dachte : "Du willst dein Lebtag nicht wieder allein vom Wege ab in den Wald laufen, wenn dir's die Mutter verboten hat. ( (1857, p. 159)

Alors tous les trois étaient heureux; le chasseur retirait la fourrure du loup et l'emportait chez lui, la grand-mère mangeait le gâteau et but le vin que Petit-bonnet-rouge lui avait apportés et se remettait de ses émotions, Petit-bonnet-rouge pensait : «Tu ne t'écarteras plus jamais du chemin pour courir seule dans la forêt, quand ta mère te l'a défendu.»

Dans le texte des Grimm, la fin est dédoublée par une procédure de relance du récit ${ }^{53}$. Rotkäppchen rencontre un jour un autre loup et montre qu'elle a retenu la leçon qu' elle avait elle-même tirée de la première histoire :

52. L'antécédent du pronom relatif est " le vin ", le gâteau n'est pas pris en compte.

53. Analysée par U. Heidmann, ouvr. cité, 20IO, p. IO7-III et ici-même. 
Es wird auch erzählt, daß einmal, als Rotkäppchen der alten Großmutter wieder Gebackenes brachte, ein anderer Wolf ihm zugesprochen und es vom Wege habe ableiten wollen. Rotkäppchen aber hütete sich und ging gerade fort seines Wegs und sagte der Großmutter, daß es dem Wolf begegnet wäre, der ihm guten Tag gewünscht, aber so bös aus den Augen geguckt hätte : "Wenn's nicht auf offner Straße gewesen wäre, er hätte mich gefressen." "Komm", sagte die Großmutter, "wir wollen die Türe verschließen, daß er nicht herein kann." Bald darnach klopfte der Wolf an und rief: "Mach auf, Großmutter, ich bin das Rotkäppchen, ich bring dir Gebackenes. » (1857, p. I59-I60)

On raconte aussi, qu'une fois, lorsque Petit-bonnet-rouge avait voulu apporter de nouveau du gâteau à sa vieille grand-mère, un autre loup lui avait adressé la parole pour la détourner de son chemin. Mais Petit-bonnet-rouge s'en était bien gardée et avait continué à aller tout droit son chemin, et elle avait dit à sa grand-mère qu'elle avait rencontré le loup, qu'il lui avait souhaité le bonjour, mais qu'il avait eu un regard si méchant : "Si ça n'avait pas été en pleine rue, il m’aurait dévorée." "Viens", dit la grand-mère, "nous allons verrouiller la porte pour qu'il ne puisse pas entrer." Peu après, le loup avait frappé à la porte et crié : «Ouvre, grand-mère, c'est Petit-bonnetrouge, je t'apporte du gâteau!»

Au lieu de reprendre cet enseignement didactiquement redoublé qui transforme le conte équivoqué mondain de Perrault en conte d'avertissement des Grimm, H. Pourrat arrête l'histoire sur la délivrance de Chaperon rouge et de sa grand-mère, et il opte pour une boucle poétique d'un distique final qui est l'écho du tout début du conte :

Effaré comme la lune rousse, le loup s'était dressé en pied. D’un seul coup de sa bonne hache, depuis le haut jusqu'en bas, du gosier au pertuis, le bûcheron lui découd tout le ventre...

Voilà qu'en sort le Chaperon rouge...

Puis tout de suite après, la mère-grand, clignant des yeux, secouant les oreilles.

- Ha, bûcheron, que tu as bien fait!

Comme c'était noir là-dedans!

N'y savait la couleur du temps! (vol. I, p. 250)

Ce distique rejoint le deuxième paragraphe du conte, en une boucle qui lie l'obscurité du bois et celle du ventre du loup : "Elles demeuraient, la vieille et la petite, au mitan d'un grand bois, tout de ramée obscure, qui était aussi noir par endroits que le ventre du loup.» (vol. I, p. 247)

\section{Analyse comparative des «Barbes bleues»}

La Barbe bleüe de Perrault diffère des trois autres textes (et de l'ensemble des Histoires ou Contes du temps passé) par l'absence de désignation générique "conte». Nous avons montré ailleurs que c'est un aspect important de sa généricité complexe d'histoire sanglante dans le style des récits de François 
de Rosset et de Jean-Pierre Camus, si célèbres dans la première moitié du $\mathrm{XVII}^{\mathrm{e}}$ siècle. Les textes des trois auteurs se ressemblent sur ce point : ils n'ont de merveilleux que la petite clé qui «était Fée» chez Perrault; la "clé défendue» du conte de Pourrat est dite magique : «Rien n'y faisait; et rien n'y pouvait faire, parce que c'était arrangé ainsi par enchantement: sur cette clé, le sang ne pouvait plus partir.» (vol. II, p. 275-276) Dans le conte des Grimm, la "petite clé en or" n'est pas dite merveilleuse, mais elle possède la même caractéristique d'être tachée de façon indélébile :

Geschwind hob sie ihn auf, und wollte das Blut abwischen, aber es war umsonst, wenn sie es auf der einen Seite abgewischt, kam es auf der andern wieder zum Vorschein; sie setzte sich den ganzen Tag hin und rieb daran, und versuchte alles Mögliche, aber es half nichts, die Blutflecken waren nicht herabzubringen; endlich am Abend legte sie ihn ins Heu, das sollte in der Nacht das Blut ausziehen. (1812, p. 287)

Rapidement, elle la ramassa et voulut essuyer le sang, mais c'était inutile, quand elle l'avait essuyé d'un côté, il réapparaissait de l'autre; elle s’assit toute la journée et frotta et essaya tout ce qu'on peut imaginer, mais il n'y avait rien à faire, les taches de sang ne pouvaient s'enlever; enfin, le soir, elle la déposa dans le foin, afin qu'il absorbe le sang durant la nuit.

C'est surtout par le rôle de l'épouse de Barbe-Bleue et par sa relation à sa famille que le conte de Pourrat tranche. Celui de Perrault dessinait la configuration d'une famille aristocratique : une «Dame de qualité» et ses "deux filles parfaitement belles», également dégoûtées par le personnage à la triste réputation, et deux frères dont nous ignorions l'existence et qui surgissaient par miracle pour abattre le tueur en série démasqué par leur sœur. Dans le conte de Pourrat, comme dans Blaubart et à la différence du texte de Perrault, la jeune femme n'a pas de sœur Anne et elle s'entretient avec ses frères, avant de se marier. Le texte des Grimm diffère de celui de Pourrat par le fait que c'est le père qui impose à sa fille ce mariage avec un roi à la barbe bleue :

Das Mädchen erschrack auch anfangs davor, und scheute sich ihn zu heiraten, aber auf Zureden ihres Vaters, willigte es endlich ein. Doch weil es so eine Angst fühlte, ging es erst zu seinen drei Brüdern, nahm sie allein und sagte "liebe Brüder, wenn Ihr mich schreien hört, wo ihr auch seid, so lasst alles stehen und liegen und kommt mir zu Hülfe». Das versprachen ihm die Brüder und küssten es, "leb wohl, liebe Schwester, wenn wir deine Stimme hören, springen wir auf unsere Pferde, und sind bald bei dir". (1812, p. 285-286)

La jeune fille s'en effrayait au début, en effet, et rechignait à l'épouser, mais, sur les exhortations de son père, elle accepta finalement. Mais parce qu'elle ressentait une telle angoisse, elle alla d'abord chez ses trois frères, les prit à part et leur dit : "Chers frères, si vous m'entendez crier, où que vous soyez, laissez tout en plan, et venez à mon secours." Ses frères le lui promirent et l'embrassèrent. «Adieu, chère sœur, quand nous entendrons ta voix, nous sauterons sur nos chevaux et serons vite chez toi.» 
Le contrat passé avec les frères est plus explicitement formulé dans le conte des Grimm que dans celui de Pourrat. Chez Pourrat, la jeune femme prend seule la décision de se marier avec la Barbe-Bleue, qui n'est pas un roi mais un simple "seigneur». Elle ne passe pas non plus de contrat d'assistance avec ses frères, car elle s'avère d'entrée fort audacieuse et courageuse :

La surveille des noces, pourtant, la fille, qu'il avait demandé pour être la septième, vit ses frères venir lui parler dans sa chambre.

«Alors, tu te maries avec la Barbe-Bleue? Tu seras bientôt prise.

- Il s'attrapera, celui qui croira me prendre!

— Pauvre petite! tu n'es pas pour longtemps sur terre!

— Frères, je vous dis, je le gagnerai!

— Va, va! il t'aura, bien plus tôt que tu ne l'auras.»

Mais elle, elle n'avait pas peur de cette barbe. (vol. II, p. 274)

Le conte de Pourrat combine les éléments du texte des Grimm et de celui de Perrault. Lors de la remise du trousseau de clé, l'effrayant mari propose, chez Pourrat et Perrault, à la jeune mariée de se divertir en son absence : "La Barbe bleüe dit à sa femme [...] qu'il la prioit de se bien divertir pendant son absence, qu'elle fit venir ses bonnes amies, qu'elle les menast à la Campagne si elle vouloit, que par tout elle fit bonne chere.» (I697, p. 6I) La jeune épouse du conte de Pourrat reçoit la même injonction : «Faites venir vos belles amies, amusez-vous comme il vous plaira : dans le château tout est vôtre» (II, p. 275), mais elle ne se livre pas à un tel divertissement mondain. À travers l'annonce de ce départ de Barbe-Bleue, elle comprend la menace et s'empresse d'écrire à ses frères une missive :

Elle pensait : «Toi, tu vas chercher ma mort.» Le tremblement la prit. Et elle courut écrire une lettre à ses frères, aussi pressante qu'elle put. Elle savait que parce qu'ils étaient ses frères, ils se mettraient tout de suite en peine de lui porter secours. (vol. II, p. 275)

À la différence des textes de Perrault et des Grimm, ce ne sont pas les frères qui tuent Barbe-Bleue : elle s'en débarrasse toute seule. Dans la chambre sanglante, les cadavres des précédentes épouses ne sont plus visibles et, en effet, pour la tuer, son époux l'emmène au bord d'un étang où, nous l'apprendrons plus tard, se trouvent les corps des précédentes victimes :

Il lui a ordonné de se dépouiller, d'abord, parce qu'il ne voulait pas voir gâter ses habits des dimanches. Mais comme il était là, devant elle, tandis qu'elle dégrafait sa robe, elle lui a dit hautement :

Ce n'est pas l'honneur d'un chevalier

De voir sa femme déshabillée!

Il n'a pu moins que de se retourner, et s'est trouvé sur le bord de l'étang; tout près des ondes. Alors, elle, sans mener aucun bruit, les deux bras en avant, elle s'approche, elle y va de sa pleine force, et d'une poussée elle le rue en plein dans l'eau. (vol. II, p. 277-278) 
C'est ainsi que périt, sans le secours des frères, le tueur en série démasqué par son épouse, aussi futée mais plus forte que ses deux consœurs. Chez Perrault, l'épouse s'empare de tous les biens amassés par l'époux monstrueux : «Il se trouva que la Barbe bleüe n'avoit point d'heritiers, \& qu'ainsi sa femme demeura maîtresse de tous ses biens.» (I697, p. 79-80) Elle en fait profiter sa sœur Anne et ses frères. Chez les Grimm, le foyer familial se reconstitue et l'épouse hérite de l'argent sale de Blaubart :

[...] die Brüder aber nahmen ihre liebste Schwester mit nach Haus, und alle Reichthümer des Blaubarts gehörten ihr. (I8I2, p. 289)

[...] Mais les frères emmenèrent leur sœur chérie à la maison, et toutes les richesses de Blaubart lui appartinrent, à elle.

La fin du conte de Pourrat est très différente. Après avoir sorti de l'étang et mis dans des cercueils les dépouilles des six premières femmes de BarbeBleue, les frères reviennent au château où se trouve leur sœur :

Cela fait, ils sont revenus chez la Barbe-Bleue. Et allez! Ils ont mis le feu partout. Tout flambait, tout craquait, tout croulait, tout volait : des fumées comme des nuages, des tourbillons d'étincelles jusqu'aux étoiles. Et avant de repartir avec leur sœur, ils l'ont regardé brûler, ce château de la Barbe-Bleue, pendant trois jours, pendant trois nuits, sans boire, ni manger, ni dormir. (vol. II, p. 278)

Il y a dans ce rituel de purification qui suit la sépulture donnée aux épouses assassinées et dans ce refus des biens maudits, une option plus radicalement religieuse et morale qui dévoile, en comparaison, cet élément étrangement absent du dénouement des deux autres récits (mais significativement développé dans Fitcher's Vogel où le personnage de Blaubart est remplacé par un sorcier).

Comme je le montre dans Textualité et intertextualité des contes ${ }^{54}$, le célèbre double dialogue de la jeune femme de Barbe bleüe avec sa sœur Anne et avec son terrible époux se présente comme une séquence fortement itérative, en quatre temps, encadrée par la reprise d'un dramatique «il faut mourir». Cet épisode joue à la fois sur la dramatisation et l'émotion, et sur l'humour et la distance ironique. La mise en italiques des énoncés répétés "Anne, ma sour Anne ne vois-tu rien venir» et «je ne voy rien que le Soleil qui poudroye, \& l'herbe qui verdoye» leur confere une valeur formulairess. Â cela s'ajoute une sophistication du genre du chant alterné qui rappelle la

54. Ibid., p. 302-308.

55. U. Heidmann a montré qu'il s'agit d'une reprise parodique des questions insistantes que la reine Didon adresse, dans L'Énéide, au moment du départ d'Énée, à sa sœur, en variant la formule "Anna, vides» (ouvr. cité, 2010, p. 139). 
forme antique du chant ou dialogue amébée (le grec amoibaios signifie «en alternance»). Cette forme se trouve aussi bien dans les parties lyriques d'une tragédie (chant alterné d'un personnage et du chœur ou de deux personnages) que dans la littérature bucolique (deux personnages se répondent par des strophes de même longueur). Je renvoie à la description que je propose de ce long dialogue, qui crée une tension dramatique par le retardement du dénouement tragique annoncé et se présente en même temps comme une parodie burlesque d'un chant alterné tragique.

La complexité de ce double chant alterné contraste avec la plus grande simplicité du passage correspondant du Blaubart des Grimm. La double communication est simplifiée. La jeune femme guette elle-même ses frères et s'adresse soit à eux, soit à son époux. Les personnages ne se répondent pas, comme c'est le cas dans le texte de Perrault. Par ailleurs, la répétition des cris-appels de la jeune femme et de son terrible mari se limite à une double triplication. Cette répétition ternaire, fréquente dans les contes des Grimm, ne se trouve pas chez Perrault qui procède à des reprises binaires et à la répétition quatre fois du même énoncé. Le montage alterné du double dialogue de La Barbe blë̈e, générateur d'un étirement du dialogue aussi dépourvu de vraisemblance que celui de la fin du Petit Chaperon rouge, se retrouve partiellement dans le Blaubart des Grimm.

Le conte de Pourrat fait également l'économie de la «sœur Anne» de Perrault et il remplace la communication quasi télépathique entre l'épouse de Blaubart et ses frères des Grimm par la lettre expédiée préalablement. Il n'est plus question non plus de prier Dieu :

Alors, il lui dit de s'habiller de ses dimanches. Elle ne se pressait pas trop, pensant bien qu'il allait la mener au bout de la galerie, comme les autres. Elle était montée dans la plus haute chambre, et à tout instant elle regardait par la fenêtre. Sitôt la lettre reçue, ses frères avaient sauté à cheval et ils étaient partis ventre à terre; mais c'était si loin! c'était si loin!... Ils ne pouvaient être là avant le soir. Et la Barbe-Bleue, qui commençait à perdre patience, criait d'en bas :

«Dévaleras-tu, ou je monte!»

- Encore un petit moment, je cherche ma plus belle robe.»

Elle tâchait d'allonger tant qu'elle pouvait, bonnes gens. Malgré elle, elle regardait si elle ne voyait pas voler la poussière des chevaux sur le chemin, mais rien ne paraissait : il n'y avait pas d'espérance. Et du bas de l'escalier la Barbe-Bleue revenait crier une fois de plus :

«Dévaleras-tu, ou je monte!»

- Encore un petit moment, je cherche ma plus belle robe.»

La minute est pourtant venue où il a fallu descendre. Il l'a amenée non pas au bout de la galerie, mais dans la salle basse. [...] (vol. II, p. 276) 
La comparaison de ce dialogue avec le texte de Perrault rend plus sensible la sophistication du chant alterné du texte de la fin du Xvir ${ }^{e}$ siècle français. La simplification du dialogue opérée par les Grimm était déjà significative, mais Pourrat va plus loin en réduisant le dialogue à un simple échange, repris une seconde fois, et il se différencie ainsi de l'intertexte de Perrault. Plus de «Sœur Anne», ni de "Soleil qui poudroye», ni d' "herbe qui verdoie", mais un souvenir de la poussière attendue des chevaux des frères guettés directement par la jeune épouse du conte des Grimm. La structure n'est pas ternaire comme chez ces derniers, mais économiquement et simplement binaire.

Le fait le plus notable est cependant la forte prise en charge énonciative marquée par la présence de discours indirect libre : «mais c'était si loin! c'était si loin!... Ils ne pouvaient être là avant le soir», et l'insertion d'un dialogue avec les lecteurs/auditeurs du conte : «bonnes gens». Cette oralité de la parole narrative est un aspect de l'écriture de Pourrat et de son historicité, comme nous l'avons vu plus haut.

\section{Conclusion épistémologique et méthodologique}

Étant donné l'intensité et la complexité de ce jeu intertextuel qui montre autant la présence des contes de Grimm que de Perrault dans l'interdiscours auvergnat de la première moitié du $\mathrm{xx}^{\mathrm{e}}$ siècle; étant donné le dialogue serré que les quatre contes modernes entretiennent avec leurs intertextes allemands et français, on peut se demander s'il est pertinent de réduire Rotkäppchen, Le Petit Chaperon rouge et Le conte du Chaperon rouge à des variantes du "conte-type " universalisé 333 "The Glutton (Red Riding Hood) " d'AarneThompson (1964, p. I25); de voir Aschenputtel, Cendrillon ou la petite pantoufle de verre et Le conte de Marie-Cendron comme des manifestations du "conte-type» 5IOA de la classification d'Aarne-Thompson (1964, p. 175). Est-il intéressant de se demander si Blaubart, La Barbe bleüe et La BarbeBleue sont des représentants du "conte-type 3 3I2 ou du "conte-type» 3II? Dans ce cas, ce ne serait plus La Barbe-Bleue de Pourrat qu'il faudrait rapprocher de Blaubart, mais Das Mordschloss (Le château aux crimes) de Front d'airain, conte de la section "Les Brigands» du Trésor des contes, et Fitcher's Vogel (L'oiseau de Fitchers) d'Isabelle et ses trois frères...

Dans la plupart des études folkloristes et littéraires, dont le livre de B. Bricout sur Le Trésor des contes, les textes sont définis par rapport à un prototype abstrait dont chaque récriture ou «transcription» constituerait une variante. 
Comme nous le montrons ailleurs ${ }^{56}$, la ressemblance des motifs a amené les folkloristes à considérer un temps L'épreuve des petits pois (Die Erbsenprobe) des Grimm ${ }^{57}$ comme une mauvaise imitation de La princesse sur le petit pois (Prindsessen paa Arten) d'Andersen. Par une analyse textuelle et comparative, nous avons mis en évidence des intrigues et des projets discursifs assez radicalement différents, reposant sur deux conceptions du genre du conte : l'ironie destructrice du conte de princes et de princesses d'Andersen n'ayant rien à voir avec la mise en valeur de la protagoniste courageuse des Grimm.

L'universalité ne permet pas de penser les spécificités, par exemple, du conte créole. Le résultat est connu : les traductions françaises des contes des Antilles passent à côté de "l'étrangeté créole des contes (Glissant dirait leur opacité), pour les installer dans une clarification conforme aux normes franco-occidentales ${ }^{58}$ ", à commencer par les normes de la langue française écrite, comme le dit si bien Patrick Chamoiseau. Ce dernier, à la différence de Pourrat, pense que l' "oraliture» des contes créoles, leur «économie orale», est profondément différente et doit être pensée comme telle dans le contexte de «la résistance nocturne, plus subtile, plus détournée, du conteur déployant sa parole au cœur même de l'habitation esclavagiste ${ }^{59}$ ». Les penseurs de la créolité nous enseignent «cette parole de rire amer contre l'Unique et le Même, riche de son propre centre et contestant tout centre, hors de toutes métropoles, et tranquillement diverselle contre l'universel ${ }^{60} »$. C'est à cette diversité négligée au profit de l'universalité que je me suis intéressé, en constituant un corpus d'une aire géographique moins lointaine, mais tout aussi particulière et historiquement située.

Le recours aux "contes-types» empêche d'entrer dans la spécificité de chaque conte et les projets d'auteurs situés socio-historiquement et esthétiquement ${ }^{6 I}$. L'approche discursive appréhende l'historicité et la singularité

56. J.-M. Adam et U. Heidmann, Le texte littéraire, 2009, p. 6I-83.

57. Conte introduit dans l'édition de I843 des Kinder-und Hausmärchen et supprimé de la sixième édition de 1850, en raison de sa trop grande ressemblance avec le conte d'Andersen (voir à ce propos J.-M. Adam et U. Heidmann, Le texte littéraire, 2009, p. 6I-83). Le même principe entraînera l'éviction de Blaubart, dès la seconde édition, parce que jugé manifestement trop proche du conte de Perrault.

58. P. Chamoiseau, "Que faire de la parole?», I994, p. I54.

59. Ibid., p. 155 .

60. P. Chamoiseau, dédicace de Chemin-d'école, Paris, Gallimard, coll. "Haute enfance», I994; cité également par U. Heidmann, qui recourt au concept de diversalité comme fondement de son approche différentielle. Voir surtout Pour une comparaison différentielle, à paraître aux Éditions Classiques Garnier.

6r. U. Heidmann le montre bien dans «Enjeux d'une comparaison différentielle et discursive. L'exemple de l'analyse des contes», 2010. 
de chaque texte, et s'intéresse aux variations socio-historiques d'un genre conte qui n'a rien de stable et de réductible à une essence. La place des contes de Perrault dans le champ de la littérature classique, le fait que Wilhelm Grimm soit un véritable écrivain, comme Henri Pourrat, tendent à disparaittre quand la piste folkloristique et une conception naïve de la littérature orale et populaire l'emportent sur l'analyse de la densité textuelle et intertextuelle de contes qui ne cessent de nous étonner par leur épaisseur sémantique ${ }^{62}$.

\section{Bibliographie}

Aarne Anti et Thompson Stith, The Types of the Folktales. A Classification and Bibliography, Helsinki, Academia Scientiarum Fennica (FFC 84), I964 [1928].

Adam Jean-Michel, La linguistique textuelle, Paris, Armand Colin, 201 .

—, "Récritures et variation : pour une génétique linguistique et textuelle», dans J.-M. Adam et I. Fenoglio (éds), Modèles linguistiques, XXX-I, vol. 9, Toulon, 2009, p. 23-50.

—, Le style dans la langue, Paris-Lausanne, Delachaux Niestlé, 1997.

Adam Jean-Michel et Goldenstein Jean-Pierre, Linguistique et discours littéraire, Paris, Larousse, 1976.

Adam Jean-Michel et Heidmann Ute, Le texte littéraire, Louvain-la-Neuve, Academia, 2009.

—, «Réarranger des motifs, c'est changer le sens. Princesses et petits pois chez Andersen et Grimm », dans A. Petitat (éd.), Contes : l'universel et le singulier, Lausanne, Payot, 2002, p. I55-174.

Bricout Bernadette, Le Savoir et la Saveur, Paris, Gallimard, 1992.

Chamoiseau Patrick, "Que faire de la parole?», dans Écrire la "parole de nuit», Paris, Gallimard, coll. "Folio/Essais n 239", I994, p. I5I-I58.

GuIssant Édouard, Introduction à une poétique du divers, Paris, Gallimard, 1996. GrÉsiLlon Almuth, «Les manuscrits littéraires : le texte dans tous ses états », Pratiques, n ${ }^{\circ}$ 57, 1988, p. I07-I22.

Grimm Jacob et Wilhelm, Kinder- und Hausmärchen, 3 vol., édition définitive, par Heinz Rölleke, Stuttgart, Philipp Reclam, 200I [I857].

-, Kinder- und Hausmärchen. Gesammelt durch die Brüder Grimm, réimpression augmentée de la première édition de I8I2 et I8I5 d'après la copie personnelle du Musée des Frères Grimm de Kassel, avec toutes

62. Merci à U. Heidmann pour sa relecture attentive de cet article et la générosité de ses conseils, son aide à la traduction, ses apports épistémologiques et sa connaissance littéraire de Perrault et des Grimm. 
les corrections manuscrites et les modifications des frères Grimm, [...] par Heinz Rölleke, Göttingen, Vandenhoeck \& Ruprecht, I986, 2 vol. [I8I2 et I8I5].

Hadjadj Dany, "À la découverte d'un atelier d'écriture : le manuscrit du Trésor des contes", Cahiers Henri Pourrat, n ${ }^{\circ}$ 6, $1988 \mathrm{a}$.

-, «Dans les marges des manuscrits d'Henri Pourrat. Quelques pistes exploratoires ", Actes du colloque La Marge, Clermont-Ferrand, Publications de l'UFR de Lettres et Sciences humaines, I988b.

Heidmann Ute, «Expérimentation générique et dialogisme intertextuel : Perrault, La Fontaine, Apulée, Straparola, Basile», Féeries, nº 8, Grenoble, Ellug, 2oII, p. 45-69.

-, «Enjeux d'une comparaison différentielle et discursive. L'exemple de l'analyse des contes", dans H. Roland et S. Vanasten (éds), Cahier voor Literatuurwetenschap, $\mathrm{n}^{\circ}$ 2, 20IO, p. 27-40.

Heidmann Ute et Adam Jean-Michel, Textualité et intertextualité des contes. Perrault, Apulée, La Fontaine, Lhéritier..., Paris, Classiques Garnier, coll. "Lire le XVII ${ }^{\mathrm{e}}$ siècle», 2010.

MeIzoz Jérôme, L'âge du roman parlant (I9I9-I939), Genève, Droz, 200I. Meschonnic Henri, La rime et la vie, Paris, Gallimard, "coll. Folio/Essais $\mathrm{n}^{\circ} 474$ ", 2006 [1989].

Perrault Charles, Histoires ou Contes du temps passé. Avec des Moralités, fac-similé du second tirage de l'édition Barbin, Paris, Firmin Didot, I929 [1697].

Philippe Gilles et Piat Julien (éds), La langue littéraire, Paris, Fayard, 2009.

Pourrat Henri, Le Trésor des contes, vol. I et II, Paris, Omnibus/Gallimard, 2009 [1948-I062].

—, Le Trésor des contes. Le bestiaire, Paris, Gallimard, 1986.

—, Le Trésor des contes. Les amours, Paris, Gallimard, coll. «Folio n ${ }^{\circ} 3857$ ", I98I [I95I].

-, Contes du vieux-vieux temps, Paris, Gallimard, coll. «Folio n 673», I970. Propp Vladimir, Morphologie du conte, Paris, Seuil, 1970 [1928].

Ramuz Charles Ferdinand, "Lettre à Bernard Grasset», dans Deux lettres, Lausanne, L'Âge d'homme, coll. "Poche suisse», I992 [1926].

Saussure Ferdinand de, «Légendes et récits d'Europe du Nord : de Sigfried

à Tristan", présentation et édition par B. Turpin, L'Herne, $\mathrm{n}^{\circ} 76$, Paris, Éditions de L'Herne, 2003, p. 35I-429.

-, Écrits de linguistique générale, Paris, Gallimard, 2002. 